1 Quantitative proteomics identifies novel PIAS1 substrates involved in cell migration and motility

Chongyang $\mathrm{Li}^{1,2}$, Francis P. McManus ${ }^{1}$, Cédric Plutoni ${ }^{1}$, Cristina Mirela Pascariu ${ }^{1}$, Trent Nelson ${ }^{1,2}$, 7 Lara Elis Alberici Delsin ${ }^{1,3}$, Gregory Emery ${ }^{1,3}$, Pierre Thibault $1,2,4,5^{*}$

$9{ }^{1}$ Institute for Research in Immunology and Cancer, ${ }^{2}$ Department of Molecular Biology, $10{ }^{3}$ Department of Pathology and Cell Biology, ${ }^{4}$ Department of Chemistry, ${ }^{5}$ Department of 11 Biochemistry, Université de Montréal, Québec, Canada

\title{
13 *Correspondence:
}

14 Pierre Thibault, Phone: (514) 343 6910, E-mail: pierre.thibault@umontreal.ca

17 Running title: Quantitative proteomics identifies novel PIAS1 substrates

Key words: SUMOylation, PIAS1, Vimentin, cell invasion, quantitative proteomics

\section{Abbreviations:}

22 SIM, SUMO-interacting motif; Ni-NTA, nickel-nitrilotriacetic acid; SCX, strong cation exchange;

23 TCE, total cell extract; DMP, dimethyl pimelimidate; TFA, trifluoroacetic acid; ACN, acetonitrile;

24 SUMO, small ubiquitin-related modifier; PML, Promyelocytic leukemia protein; PTM, post25 translational modification; LC-MS/MS, liquid chromatography-tandem mass spectrometry; FDR, 26 false discovery rate; PIAS1, Protein Inhibitor of Activated STAT, 1; SENPS, SUMO specific 27 proteases; VIM, Vimentin; ULF, Unit-length filament; VIFs, Vimentin Intermediate Filaments; 


\section{ABSTRACT}

30 The Protein Inhibitor of Activated STAT 1 (PIAS1) is an E3 SUMO ligase that plays important roles

31 in various cellular pathways, including STAT signaling, p53 pathway, and the steroid hormone

32 signaling pathway. PIAS1 can SUMOylate PML (at Lys-65 and Lys-160) and PML-RAR $\alpha$ promoting

33 their ubiquitin-mediated degradation. Increasing evidence shows that PIAS1 is overexpressed in

34 various human malignancies, including prostate and lung cancers. To understand the mechanism

35 of action of PIAS1, we developed a quantitative SUMO proteomic approach to identify potential

36 substrates of PIAS1 in a system-wide manner. Our analyses enabled the profiling of 983 SUMO

37 sites on 544 proteins, of which 204 SUMO sites on 123 proteins were identified as putative PIAS1

38 substrates. These substrates are involved in different cellular processes, such as transcriptional

39 regulation, DNA binding and cytoskeleton dynamics. Further functional studies on Vimentin

40 (VIM), a type III intermediate filament protein involved in cytoskeleton organization and cell

41 motility, revealed that PIAS1 exerts its effects on cell migration and cell invasion through the

42 SUMOylation of VIM at Lys-439 and Lys-445 residues. VIM SUMOylation was necessary for its

43 dynamic disassembly, and cells expressing a non-SUMOylatable VIM mutant showed reduced

44 levels of proliferation and migration. Our approach not only provides a novel strategy for the

45 identification of E3 SUMO ligase substrates, but also yields valuable biological insights into the

46 unsuspected role of PIAS1 and VIM SUMOylation on cell motility. 


\section{INTRODUCTION}

The small ubiquitin-like modifier (SUMO) protein is an ubiquitin-like (UBL) protein that is highly dynamic and can reversibly target lysine residues on a wide range of proteins involved in

51 several essential cellular events, including protein translocation and degradation, mitotic

52 chromosome segregation, DNA damage response, cell cycle progression, cell differentiation and

53 apoptosis ${ }^{1}$. SUMO proteins are highly conserved through evolution, and the human genome

54 encodes 4 SUMO genes, of which 3 genes (SUMO-1, SUMO-2 and SUMO-3) are ubiquitously 55 expressed in all cells ${ }^{1,2}$. Prior to conjugation, the immature SUMO proteins are C-terminally 56 processed by sentrin-specific proteases (SENPs) ${ }^{3}$. These proteases also cleave the isopeptide

57 bond formed between the $\varepsilon$-amino group of the acceptor lysine residues and the C-terminus 58 residue of the conjugated SUMO proteins. The conjugation of SUMO to target proteins requires 59 an E1 activating enzyme (SAE1/2), an E2 conjugating enzyme (UBC9) and one of several E3 SUMO

60 ligases ${ }^{4}$. Unlike ubiquitination, in vitro SUMOylation can occur without E3 SUMO ligases, although 61 enhanced substrate specificity is conferred by E3 SUMO ligases ${ }^{5}$. It is believed that SUMOylation 62 events occurring without the aid of E3 SUMO ligases arise primarily on the consensus motif 63 composed of $\psi \mathrm{KxE}$, where $\psi$ represents a large hydrophobic residue and $x$, any amino acid ${ }^{6}$. To 64 date, several structurally unrelated classes of proteins appear to act as E3 SUMO ligases in 65 mammalian cells, such as the protein inhibitor of activated STAT (PIAS) family of proteins, Ran66 binding protein 2 (RanBP2), the polycomb group protein (Pc2), and topoisomerase I- and p5367 binding protein (TOPORS) $7,8$.

PIAS orthologs can be found through eukaryote cells, and comprise four PIAS proteins (PIAS1, PIASx (PIAS2), PIAS3, and PIASy (PIAS4)) that share a high degree of sequence homology ${ }^{9}$. Overall, five different domains or motifs on PIAS family proteins recognize distinct sequences or

71 conformations on target proteins, unique DNA structures, or specific "bridging" molecules to

72 mediate their various functions ${ }^{10}$. An example of this is the PIAS Scaffold attachment factor (SAP)

73 domain which has a strong affinity towards A-T rich DNA ${ }^{11}$ and binds to Matrix attachment

74 regions $D_{N A}^{12}$, in addition to having an important role in substrate recognition ${ }^{13}$. The PINIT motif 75 affects subcellular localization and contributes to substrate selectivity ${ }^{14,15}$. The Siz/PIAS RING (SP76 RING) domain interacts with UBC9 and facilitates the transfer of SUMO to the substrate ${ }^{16}$. The 
PIAS SIM (SUMO interaction motif) recognizes SUMO moieties of modified substrates and alters subnuclear targeting and/or assembly of transcription complex ${ }^{16-18}$. While several functions have been attributed to these domains, relatively little is known about the role of the poorly conserved C-terminus serine/threonine-rich region.

PIAS1 is one of the most well studied E3 SUMO ligases, and was initially reported as the 82 inhibitor of signal transducers and activators of transcription 1 (STAT1) ${ }^{19}$. Previous studies 83 indicated that PIAS1 interacts with activated STAT1 and suppresses its binding to DNA ${ }^{8}$. PIAS1 84 overexpression was reported in several cancers, including prostate cancer, multiple myeloma, 85 and B cell lymphomas ${ }^{20-23}$. PIAS1 can SUMOylate the Focal adhesion kinase (FAK) at Lys-152, a 86 modification that dramatically increases its ability to autophosphorylate Thr-397, activate FAK, 87 and promotes the recruitment of several enzymes including Src family kinases ${ }^{24}$. In yeast, Lys-164 88 SUMOylation on Proliferating Cell Nuclear Antigen (PCNA) is strictly dependent on the PIAS1 89 ortholog Siz1, and is recruited to the anti-recombinogenic helicase Srs2 during S-phase ${ }^{25}$. PIAS1 can also regulate oncogenic signaling through the SUMOylation of promyelocytic leukemia (PML)

91 and its fusion product with the retinoic acid receptor alpha (PML-RAR $\alpha$ ) as observed in acute 92 promyelocytic leukemia $(\mathrm{APL})^{26}$. In addition to its regulatory role in PML/ PML-RAR $\alpha$ oncogenic 93 signaling, PIAS1 has been shown to be involved in the cancer therapeutic mechanism of arsenic 94 trioxide (ATO). This is accomplished by ATO promoting the hyper-SUMOylation of PML-RAR $\alpha$ in 95 a PIAS1-dependent fashion, resulting in the ubiquitin-dependent proteasomal degradation of 96 PML-RAR $\alpha$ and APL remission ${ }^{26}$. In B cell lymphoma, PIAS1 has been reported as a mediator in 97 lymphomagenesis through SUMOylation of MYC, a proto-oncogene transcription factor 98 associated with several cancers. SUMOylation of MYC leads to a longer half-life and therefore an 99 increase in oncogenic activity ${ }^{23}$. Altogether, these reports suggest that PIAS1 could promote 100 cancer cell growth and progression by regulating the SUMOylation level on a pool of different 101 substrates.

102 In this study, we first evaluate the effect of PIAS1 overexpression in HeLa cells. PIAS1 103 overexpression has a significant influence on cell proliferation, cell migration and cancer cell 104 invasion. To identify putative PIAS1 substrates, we developed a system level approach based on 
105 quantitative SUMO proteomic analysis ${ }^{27}$ to profile changes in protein SUMOylation in cells 106 overexpressing this E3 SUMO ligase. Our findings revealed that 204 SUMO sites on 123 proteins 107 were regulated by PIAS1. Bioinformatic analysis indicated that many PIAS1 substrates are 108 involved in transcription regulation pathways and cytoskeleton organization. Interestingly, 109 several PIAS1 substrates, including cytoskeletal proteins (Actin filaments, Intermediate filaments 110 and Microtubules), were SUMOylated at lysine residues located in non-consensus motif. We 111 confirmed the SUMOylation of several PIAS1 substrates using both in vitro and in vivo 112 SUMOylation assays. Further functional studies revealed that PIAS1 mediated the SUMOylation 113 of vimentin (VIM) at two conserved sites on its C-terminus that affect the dynamic disassembly 114 of this intermediate filament protein. 


\section{RESULTS}

PIAS1 Overexpression Promotes cell Proliferation and Motility

118 To investigate the physiological function of PIAS1 in HeLa cells, we overexpressed PIAS1 and

119 evaluated its expression level by western blot. The abundance of PIAS1 in HeLa cells was

120 increased by 2.5 fold at $48 \mathrm{~h}$ post-transfection (Fig. 1a). PIAS1 overexpression promotes HeLa cell

121 proliferation by $\sim 50 \%$ (Fig. 1b). We further examined the phenotypic effects of PIAS1

122 overexpression on cell migration and cell invasion using wound-healing and Boyden chamber

123 invasion assays. The migration and invasion ability of Hela cells were both increased after PIAS1

124 overexpression (Figs. 1c-f). Taken together, these results highlight the role that PIAS1 plays in

125 regulating cell growth, cell cycle, cell migration and cell invasion of HeLa cells.

128 To gain a better understanding of the role that PIAS1 plays in cell cycle regulation, cell 129 proliferation, invasion and motility, we devised a large-scale SUMO proteomic approach to 130 identify PIAS1 substrates in a site-specific manner (Fig. 2). We combined a SUMO remnant 131 immunoaffinity strategy ${ }^{27}$ with metabolic labeling (stable isotope labeling of amino acid in cell 132 culture, SILAC) to study the global changes in protein SUMOylation upon PIAS1 overexpression. 133 HEK293 cells stably expressing SUMO3m (Supplementary Fig. 1) were grown at $37{ }^{\circ} \mathrm{C}$ in media 134 containing light ( $\left.{ }^{0} \mathrm{Lys},{ }^{0} \mathrm{Arg}\right)$, medium $\left({ }^{4} \mathrm{Lys},{ }^{6} \mathrm{Arg}\right)$, or heavy $\left({ }^{8} \mathrm{Lys},{ }^{10} \mathrm{Arg}\right)$ isotopic forms of lysine 135 and arginine. Three biological replicates were performed, and for each replicate, one SILAC 136 channel was transfected with an empty vector while the other two were transfected with Myc137 PIAS1 vectors (Fig. 2a). At $48 \mathrm{~h}$ post-transfection, an equal amount of cells from each SILAC 138 channel were harvested and combined before lysis in a highly denaturant buffer. PIAS1 139 overexpression efficiency in HEK293 SUMO3m cells was evaluated by western blot (Fig. 2b). 140 Protein extracts were first purified by Ni-NTA beads to enrich SUMO-modified proteins and 141 digested on beads with trypsin (Fig. 2c). Following tryptic digestion, SUMO-modified peptides 142 were immunopurified using an antibody directed against the NQTGG remnant that is revealed on 
143 the SUMOylated lysine residue. Next, peptides were fractionated by offline strong cation

144 exchange (SCX) STAGE tips and analyzed by LC-MS/MS on a Tribrid Fusion instrument. To

145 determine that abundance changes were attributed to SUMOylation and not to change in protein

146 expression, we also performed quantitative proteomic analyses on the total cell extracts from

147 PIAS1 overexpression (Fig. 3a and Supplementary Fig. 2). PIAS1 overexpression caused a global

148 increase in protein SUMOylation with negligible changes on protein abundance (Fig. 3b). In total,

14912080 peptides on 1756 proteins (Fig. 3a, Supplementary Table 1) and 983 SUMO peptides on

150544 SUMO proteins (Fig. 3b, Supplementary Table 2) were quantified for the proteome and

151 SUMO proteome analyses, respectively. A total of 204 SUMOylation sites on 123 proteins were

152 found to be upregulated by PIAS1 overexpression including known substrates such as PCNA and

153 PML. A summary of these analyses is shown in Fig. 3 c.

154 Protein classification ontology analysis of the PIAS1 substrates using PANTHER clustered the 155 targets into 15 groups (Fig. 3d). PIAS1 mediated SUMOylation predominantly occurred on nucleic 156 acid binding proteins, transcription factors, cytoskeletal proteins, enzyme modulators, ligases 157 and chaperone proteins. We next classified putative PIAS1 substrates by their gene ontology (GO) 158 molecular function, biological process and cellular components (Fig. 3e) using the whole 159 identified SUMOylome as background. GO cellular component classification revealed that PIAS1 160 substrates were enriched in the plasma membrane and cytosol compared to the global 161 SUMOylome (Fig. 3e). This suggests that PIAS1 substrates may undergo nucleocytosolic shuttling 162 upon SUMOylation. GO biological process analysis revealed that identified PIAS1 substrates are 163 involved in a variety of biological processes, such as protein stabilization, protein folding, histone $164 \mathrm{H} 3$ acetylation, translational initiation, and signal recognition particle (SRP)-dependent co165 translational protein targeting to membrane (Fig. 3e). GO molecular function analysis indicated 166 that PIAS1 substrates are associated with ubiquitin protein ligase binding, ubiquitin-protein 167 transferase activity and unfolded protein binding (Fig. 3e). Indeed, PIAS1 regulates the 168 SUMOylation of several proteins whose roles in the cell are diverse. Much like global 169 SUMOylation, PIAS1 mediated SUMOylation may play a role in several biological processes that 170 are independent from each other. 
171 Previous SUMO proteome analyses indicated that under unstressed conditions,

172 approximately half of acceptor lysine residues are found in the SUMO consensus and reverse

173 consensus motifs ${ }^{28}$. Since it is believed that SUMOylation that occurs on the consensus motif

174 does not absolutely require an E3 ligase, we surmised that PIAS1 mediated SUMOylation may

175 occur at non-consensus motifs. We therefore compared the amino acid residues surrounding the

176 SUMOylation sites that are regulated by PIAS1 to those of the whole SUMO proteome

177 (Supplementary Fig. 3a). As anticipated, the sequences surrounding the PIAS1 mediated

178 SUMOylation sites are depleted in glutamic acid at position +2 and depleted of large hydrophobic

179 amino acids at position -1, consistent with the reduction of the consensus sequence. Indeed, E3

180 SUMO ligases appear to aid in the SUMOylation of lysine residues that reside in non-canonical

181 regions. Furthermore, we investigated the local secondary structures and solvent accessibility of

182 PIAS1 substrates surrounding SUMO sites using NetSurfP-1.1 software (Supplementary Fig. 3b).

183 We observed that one-third of PIAS1 regulated SUMOylation sites are located within $\alpha$-helix and

184 approximately one-tenth within $\beta$-strand. In contrast, the majority of SUMOylated lysine residues

185 in the SUMO proteome are localized in coil regions. Taken together, these results support the

186 notion that PIAS1 mediated SUMOylation preferentially occurs on structured regions of the

187 protein, which may help substrate recognition by PIAS1. Additionally, we noted that PIAS1

188 mediated SUMOylation occurred primarily on solvent exposed lysine residues, which was also

189 the case for the global SUMOylome. These results suggest that PIAS1 may not impart

190 conformational changes to its substrate upon binding since it does not promote SUMOylation on

191 lysine residues that would otherwise be buried within the core of the substrate. Overall, PIAS1

192 promotes the ability of UBC9 to SUMOylate lysine residues that are present in non-consensus

193 sequences located on ordered structures of the proteins.

194 To better understand the cellular processes regulated by PIAS1, a STRING analysis was

195 performed to analyze the interaction network of putative PIAS1 substrates. This network

196 highlights the presence of highly connected interactors from promyelocytic leukemia protein

197 (PML) nuclear body, transcriptional factors, cytoskeletal proteins and RNA binding proteins (Fig.

198 4). PIAS1 was previously shown to colocalize to PML nuclear body and to regulate oncogenic 
signaling through SUMOylation of PML and its gene translocation product PML-RAR $\alpha$ associated with acute promyelocytic leukemia (APL) ${ }^{26}$.

201 Interestingly, we also found that several putative PIAS1 substrates were associated with 202 cytoskeletal organization including $\beta$-actin (ACTB), $\alpha$-tubulin (TUBA1B), vimentin (VIM) and 203 Keratin 8 (KRT8), in addition to several other intermediate filament proteins. Of note, Keratin 8 204 was reported to be SUMOylated in vitro and in vivo, a modification that changes its dynamic 205 assembly and is increased in cells and tissues during apoptosis, oxidative stress, and phosphatase 206 inhibition ${ }^{29}$. The actin filaments, intermediate filaments, and microtubules that form the 207 cytoskeleton of eukaryotic cells are responsible for cell motility and division. They also help establish cell polarity, which is required for cellular homeostasis and survival ${ }^{30}$. Moreover, one 209 SUMO site on $\beta$-actin (ACTB) and five SUMO sites on $\alpha$-tubulin (TUBA1B) were found at non210 consensus motif regions, indicating an important role for PIAS1 in substrate protein dynamics

211 during SUMOylation. Additionally, we evaluated the degree of evolutionary conservation of these 212 modified lysine residues. Surprisingly, all SUMOylated lysine residues analyzed are highly 213 conserved across different species (Supplementary Figs. 4 and 5). In our data, we found 214 intermediate filaments (IFs) to be major targets of PIAS1 among cytoskeletal proteins. Our results 215 highlight that PIAS1 mediates the SUMOylation of the type II IF keratin 8 protein at Lys-421 and 216 the type III IF VIM protein at Lys-439 and Lys-445, both on their respective tail domains. 217 Moreover, PIAS1 promotes the SUMOylation of several type V IF proteins (e.g. Prelamin A/C, 218 Lamins B1 and B2) on their Rod domains (Supplementary Fig. 6).

\section{Validation of identified PIAS1 substrates by in vitro and in vivo SUMOylation assays}

221 Next, we selected E3 SUMO-protein ligase NSE2 (NSMCE2), prefoldin subunit 2 (PFDN2) and 222 VIM, which were identified in SUMO proteomic experiments as putative PIAS1 substrates for 223 further validation. We performed both in vitro and in vivo SUMOylation assays to confirm that 224 these sites were regulated by PIAS1. For the in vitro SUMO assay, we incubated individual SUMO 225 substrates with SUMO-activating E1 enzyme (SAE1/SAE2), UBC9, SUMO-3 with or without PIAS1 in the presence of ATP. We also used PCNA, a known PIAS1 substrate, as a positive control. After 
$2274 \mathrm{~h}$ incubation at $37^{\circ} \mathrm{C}$, the western blots of each substrate showed either single or multiple bands

228 of higher molecular weight confirming the SUMOylated products. Separate LC-MS/MS

229 experiments performed on the tryptic digests of the in vitro reactions confirmed the

230 SUMOylation of NSMCE2 at residues Lys-90, Lys-107, and Lys-125, and PFDN2 at residues Lys-94,

231 Lys-111, Lys-132, and Lys-136. While UBC9 alone can SUMOylate these substrates, we noted an

232 increasing abundance of SUMOylated proteins when PIAS1 was present, confirming that the E3

233 SUMO ligase enhanced the efficiency of the conjugation reaction (Figure S7a). Interestingly,

234 several SUMOylation sites that were regulated by PIAS1 on both NSMCE2 and PFDN2 were not

235 located within SUMO consensus motifs, further supporting the motif analysis of the large-scale

236 proteomic data (Supplementary Fig. 3a).

237 Furthermore, we examined whether PIAS1 contributes to substrate SUMOylation in vivo. 238 HEK293-SUMO3 cells were co-transfected with Flag-NSMCE2, PFDN2 or VIM and Myc-PIAS1. Co-

239 transfected cells were subjected to immunoprecipitation with anti-Flag agarose gel, followed by 240 western blot with an anti-His antibody. The SUMOylation of substrates was minimally detected

241 when only transfecting Flag-substrates. In contrast, overexpression of PIAS1 under the same 242 experimental conditions led to a marked increase in the SUMOylation of these substrates 243 (Supplementary Fig. 7b). These results further confirm our quantitative SUMO proteomics data 244 and validate the proteins NSMCE2, PFDN2 and VIM as bona fide PIAS1 substrates.

\section{PIAS1 SUMOylation Promotes its Localization to PML-NBS}

247 Interestingly, our large-scale SUMO proteomic analysis identified five SUMOylation sites on 248 PIAS1 (Fig. 5a). Two of these sites (Lys-46, Lys-56) are located in the SAP domain, and may 249 regulate the interaction of PIAS1 with DNA ${ }^{15}$. We also identified two SUMOylated residues (Lys250137 and Lys-238) located within the PINIT domain of PIAS1, potentially affecting its subcellular 251 localization ${ }^{14}$. The last SUMOylated site (Lys-315) of PIAS1 and is located next to an SP-RING 252 domain, which may alter the ligation activity of PIAS1 ${ }^{31}$. Of note, PIAS1 contains a SUMO 253 interaction motif (SIM), and previous reports indicated that this ligase can localize to PML nuclear 254 bodies in a SIM-dependent manner with SUMOylated PML ${ }^{32}$. As PML also contains a SIM motif, 
255 we were interested in three out of the five SUMO sites on PIAS1: Lys-137, Lys-238 and Lys-315.

256 Since PIAS1 is SUMOylated at several sites and PML contains a SIM, we surmised that reciprocal

257 interactions could be mediated through SUMO-SIM binding. Accordingly, we constructed a

258 PIAS1-GFP vector and used site-directed mutagenesis to specifically mutate the PIAS1 lysine

259 residues that are SUMO modified and are located within regions of PIAS1 that could interact with

260 PML. As the SAP domain of PIAS1 is exclusively reserved for DNA binding, we excluded the SUMO-

261 modified lysine residues in this domain when creating the mutant construct as it may affect its

262 localization in a PML independent fashion. We therefore created the variant constructs by

263 mutating the codons for Lys-K137, Lys-238 and Lys-315 to arginine codons. Several mutant genes

264 were created, including single mutants of each site, double mutant combinations, and the triple

265 mutant (PIAS1-GFP 3xKR). These mutant vectors were transfected into HEK293 SUMO3m cells

266 and used to study the effects of SUMOylation at the various lysine residues on the PIAS1-PML

267 colocalization. As evidenced by the immunofluorescence studies, approximately $45 \%$ of WT

268 PIAS1-GFP colocalized with PML (Fig. 5b). No significant changes in the colocalization of PIAS1

269 and PML were observed when experiments were repeated using either single or double PIAS1-

270 GFP mutants (data not shown). However, we noted a 50\% reduction in PIAS1-GFP-PML

271 colocalization when all three sites were mutated, suggesting a possible functional redundancy

272 among these sites (Figs. 5b and 5c). The fact that the co-localization of PML and PIAS1-GFP was

273 not totally abrogated for the triple mutant might be explained by residual interactions between

274 SUMOylated PML and the SIM of PIAS1-GFP32. Taken together, these experiments confirmed that

275 colocalization of PIAS1 at PML nuclear bodies is partly mediated by the SUMOylation of PIAS1 at

276 Lys-137, Lys-238 and Lys-315 residues.

279 VIM is predominantly found in various mesenchymal origins and epithelial cell lines ${ }^{33-35}$. 280 Increasing evidence shows that VIM plays key roles in cell proliferation ${ }^{36}$, migration ${ }^{37}$ and 281 contractility ${ }^{38}$. Our data shows that two SUMO sites on VIM are regulated by PIAS1, both of which 282 are located on the tail domain and are highly conserved across different species (Fig. 6a). To 
283 further investigate the function of PIAS1 mediated SUMOylation of VIM, we expressed a Flagtagged VIM K439/445R double mutant (VIM ${ }^{\mathrm{mt}}$ ) that is refractory to SUMOylation in HeLa cells, and compared the functional effects to cell expressing the wild-type Flag-tagged VIM (VIM $\left.{ }^{w t}\right)$. We transfected Flag-VIM ${ }^{\text {wt }}$ and Flag-VIM ${ }^{\text {mt }}$ into HeLa cells and used the empty Flag vector as a negative control. At $48 \mathrm{~h}$ post-transfection, the cells were harvested, lysed in $8 \mathrm{M}$ urea and protein pellets were separated on SDS-PAGE. The ensuing western blot results show that protein abundance between $\mathrm{VIM}^{\mathrm{wt}}$ and $\mathrm{VIM}^{\mathrm{mt}}$ are similar; yet, the SUMO level on $\mathrm{VIM}^{\mathrm{mt}}$ is undetectable

290 (Fig. 6b). Growth assays demonstrate that VIM ${ }^{\text {wt }}$ expression has no effect on HeLa cell 291 proliferation while $\mathrm{VIM}^{\mathrm{mt}}$ expression results in a significant inhibition of cell growth (Fig. 6c).

292 Moreover, we examined the effect of $\mathrm{VIM}^{\mathrm{wt}}$ and $\mathrm{VIM}^{\mathrm{mt}}$ expression on cell migration and invasion 293 using wound-healing and Boyden chamber invasion assays. VIM ${ }^{\text {wt }}$ significantly promotes cell 294 migration and invasion, which is in line with the results obtained in HepG2 cells ${ }^{39}$. However, $295 V I_{M}^{m t}$ conferred no effect on cell migration or invasion (Figs. 6d-g). These results indicate that 296 SUMOylation of VIM plays a role in cell growth, migration and invasion, presumably by regulating 297 VIM IF function and/or formation.

298 Next, we investigated the function of VIM SUMOylation on the dynamics assembly of IFs. Both 299 Keratin and lamin A IFs formation and solubility have been reported to be regulated by 300 SUMOylation, while such properties have yet to be uncovered for VIM IFs ${ }^{40}$. For example, the 301 SUMOylation of lamin A at Lys-201, which is found in the highly conserved rod domain of the 302 protein, results in its proper nuclear localization ${ }^{41}$. Unlike lamin A SUMOylation, keratin 303 SUMOylation is not detected under basal conditions. However, stress-induced keratin 304 SUMOylation has been observed in mouse and human in chronic liver injuries. Additionally, 305 keratin monoSUMOylation is believed to increase its solubility, while hyperSUMOylation 306 promotes its precipitation ${ }^{42}$.

307 We surmised that SUMOylation on VIM would alter its solubility, akin to the properties 308 observed for keratin. Accordingly, we transfected an empty vector as a negative control, VIM ${ }^{\mathrm{wt}}$ 309 and VIM ${ }^{m t}$ into HeLa cells and lysed the cells with a RIPA buffer. Samples were fractionated into 310 RIPA soluble and insoluble fractions. Western blot analysis of these samples shows that VIM ${ }^{\text {wt }}$ is 
311 preferentially located in the RIPA soluble fraction, while VIM ${ }^{\text {mt }}$ resides more in the insoluble

312 fraction (Fig. 7a). Clearly, SUMOylation of VIM drastically increases its solubility.

313 IF networks are subject to regulation by a number of phosphatases and kinases ${ }^{43}$. Stable VIM

314 filaments undergo extensive reorganization upon phosphorylation, a posttranslational

315 modification that controls VIM assembly and disassembly ${ }^{44-46}$. Multiple phosphorylation sites

316 have now been identified on $\mathrm{VIM}^{47}$. Those that induce disassembly are located in the $\mathrm{N}$-terminal

317 head domain and are phosphorylated by multiple protein kinases. Phosphorylation of the head

318 region increases the distance between the two head groups of the VIM dimer, thus rendering the

319 VIM tetramer incapable of assembling into a filament. Activation of Akt in soft-tissue sarcoma

320 (STS) cells promotes the interaction of the head region of VIM and the tail region of Akt, resulting

321 in the phosphorylation of VIM at Ser-39, further enhancing cell motility and invasion ${ }^{41}$.

322 Next, we sought to examine if VIM SUMOylation alters its phosphorylation status, which 323 could lead to changes in VIM IF dynamic assembly/disassembly. Accordingly, we separated 324 protein extracts from VIM ${ }^{w t}$ and VIM $^{m t}$ by SDS-PAGE, excised bands that corresponded to the 325 soluble and insoluble VIM, and performed in-gel trypsin digestion followed by LC-MS/MS 326 (Supplementary Fig. 8). We identified several phosphorylated serine residues and one 327 phosphorylated threonine residue on VIM (Supplementary Table 3), which have also been 328 reported in the literature (S5, S7, T20, S22, S26, S29, S39, S42, S51, S56, S66, S72, S73, S83, S226, 329 T258 and S459). All sites except those located on the C-terminal were found to be 330 hyperphosphorylated in the insoluble $\mathrm{VIM}^{\mathrm{mt}}$ compared to the wild-type counterpart. 331 Interestingly, we observed an increase phosphorylation of S39 in the insoluble pellet of VIM ${ }^{\mathrm{mt}}, \mathrm{a}$ 332 site known to be phosphorylated by Akt ${ }^{41}$. The observation that VIM is hyperphosphorylated at 333 its $\mathrm{N}$-terminus in VIM $^{\mathrm{mt}}$ suggests a possible cross-talk between SUMOylation and 334 phosphorylation.

335 To further understand how SUMOylation affects VIM dynamics in vivo, we transfected 336 Emerald-VIM ${ }^{\text {wt }}$ or $-\mathrm{VIM}^{\mathrm{mt}}$ vectors in VIM null MCF-7 cells. VIM null cells were employed to 337 eliminate the contribution of endogenous VIM on the VIM dynamics, which could mask the 338 phenotypic effects of our mutant. Using fluorescence microscopy we quantified the proportion 
of the various VIM structures for the two different Emerald tagged constructs. We found three major forms of VIM in the cells, which in accordance with the literature, were categorized as

341 cytosolic, unit-length filament (ULF) and VIM intermediate filaments (VIFs) (Supplementary Fig.

342 9). Statistical analysis of the proportion of the VIM structures revealed that the SUMO 343 conjugation deficient VIM (VIM ${ }^{\mathrm{mt}}$ ) promoted the formation of ULF with a concomitant reduction

344 in VIF formation compared to its wild-type counterpart (Fig. 7b). Taken together these results

345 indicate that SUMOylation of VIM promotes the formation of VIFs from the ULF building blocks.

346 Although VIM filament growth primarily relies on elongation by the longitudinal annealing of

347 ULFs via end-to-end fusion, recent studies suggest that the subunit exchange of tetramers within 348 these filaments does occur ${ }^{48}$. To determine if the SUMOylation of VIM affects the subunit 349 exchange rate of these filaments we performed fluorescence recovery after photobleaching 350 (FRAP) assays (Supplementary Fig. 10). We monitored the recovery time of filament fluorescence 351 up to $300 \mathrm{~s}$ after bleaching for both Emerald-VIM ${ }^{\text {wt }}$ and Emerald-VIM ${ }^{\mathrm{mt}}$, and noted no statistical 352 difference in recovery between constructs (Fig. 7c). This observation suggests that the 353 SUMOylation of VIM is not involved in the subunit exchange of tetramers within filaments.

354 The model described in Fig. 7d combines the results from the proteomic, 355 immunofluorescence and FRAP assays and describes the molecular mechanism of PIAS1 356 mediated control of VIM dynamics. Under physiological conditions, vimentin is maintained in 357 equilibrium between ULF and soluble tetramers. The formation of VIM ULF has been shown to 358 occur spontaneously on the order of seconds in vitro showing that this arrangement is 359 thermodynamically favorable and proceeds rapidly without the need for protein modifications ${ }^{49}$. 360 Vimentin filaments elongate by end-to-end annealing of ULFs and eventually form mature 361 vimentin intermediate filaments (VIFs). This is followed by the breakdown of VIFs by severing, 362 which involves phosphorylation on several residues found on the $\mathrm{N}$-term of $\mathrm{VIM}^{47,50}$. We show in 363 this work that this hyper-phosphorylation occurs exclusively on the N-terminal of VIM in a SUMO364 dependent mechanism. The truncated filaments can reanneal with another ULF to form larger 365 VIFs. However, the phosphorylated ULF must be SUMOylated by PIAS1 to increase either the 366 solubility or interaction with protein phosphatases, such as type-1 (PP1) and type-2A (PP2A) 
367 protein phosphatases, as shown by the large increase in vimentin phosphorylation levels with 368 the SUMO deficient vimentin construct. ${ }^{47}$ These results suggest that the PIAS1-mediated 369 SUMOylation of VIM stimulates the dephosphorylation of ULF and facilitate the reentry of the 370 ULF into the VIF maturation process by annealing on growing VIFs. This dynamic assembly and 371 disassembly of VIFs thus involve the SUMOylation of VIM, a modification that also regulates the 372 cell proliferation, migration and invasion (Fig. 6). 


\section{DISCUSSION}

375 We report the functional effect of E3 SUMO ligase PIAS1 in HeLa cells, and determined that 376 PIAS1 not only promotes cell proliferation, but also stimulates cell migration and invasion. PIAS1 377 has been extensively studied in other cancer lines, such as Human Prostate Cancer, where PIAS1 378 expression is increased and enhanced proliferation through inhibition of $\mathrm{p} 21^{21}$. In addition, other 379 studies have also reported that PIAS1 may function as a tumor suppressor to regulate gastric 380 cancer cell metastasis by targeting the MAPK signaling pathway ${ }^{51}$. IL-11 mediated decrease in 381 HTR-8/SVneo cells invasiveness was associated with a decrease in ERK1/2 activation, PIAS1/3382 mediated activated STAT3 (Tyr-705) sequestration, and a decrease in PIAS1 expression, leading 383 to a decrease in the expression of Fos and major families of metalloproteinase (MMP2, MMP3, 384 MMP9 and MMP23B) ${ }^{52}$. However, these studies were limited to individual PIAS1 targets to 385 understand the regulation mechanism. These targeted approaches sufficed to answer specific 386 questions about PIAS1 mediated SUMOylation, but lack the depth to fully elucidate the function 387 of PIAS1. A systematic approach to establish the global properties of PIAS1 as an E3 SUMO ligase 388 and how these SUMOylation events alter substrate function are missing and needed. Indeed, 389 such a method was never conceived due to the complex nature of quantitative SUMO 390 proteomics. Global SUMO proteome analyses are challenging due to the low abundance of 391 protein SUMOylation and the extremely large remnant that is retained on the modified lysine 392 residues upon tryptic digestion. Moreover, proteomic workflows that are currently available to 393 study SUMOylation require two levels of enrichment, which adversely affects the reproducibility 394 of SUMO site quantitation.

395 We devised an efficient method for the identification PIAS1 substrates by modifying our 396 previously described SUMO proteomics approach ${ }^{27}$. This method combines SILAC labelling for 397 reproducible quantitative proteomic analyses, E3 SUMO ligase protein overexpression, followed 398 by SUMO remnant immunoaffinity enrichment. This workflow allows for the selective profiling 399 of substrates and regulated SUMOylation sites of any E3 SUMO ligase. All the PIAS1 substrates 400 identified in this work were analyzed using forward and reverse SILAC labeling under basal 401 condition, which further increases the confidence of the identified substrates. Notably, we 
402 observed that PIAS1 overexpression has a global effect on protein SUMOylation (Fig. 3b). This is

403 in part due to some PIAS1 substrates being directly involved in protein SUMOylation, such as

404 PIAS2, PIAS3, NSMCE2, TOPORS and TRIM28. In addition, many of the identified substrates were

405 found to participate in protein ubiquitination regulation, such as TRIM24, TRIM33, RNF2 and

406 BRCA1, which may also affect the global protein SUMOylation through the interplay between

407 SUMOylation and ubiquitination 27,53 .

408 We identified five SUMOylation sites on PIAS1 itself (Lys-46, Lys-56, Lys-137, Lys-238 and Lys409 315), suggesting a possible feedback mechanism that could keep SUMOylation levels in check.

410 Our immunofluorescence studies show that SUMOylation of PIAS1 promotes its localization to 411 PML nuclear bodies (Figs. 5c and 5d). Interestingly, a recent paper that studied the substrates of

412 RNF4 identified PIAS1 as a substrate of this SUMO-targeted ubiquitin ligase ${ }^{54}$. Moreover, RNF4 is 413 localized to PML nuclear bodies, where it ubiquitylates SUMOylated proteins for their 414 subsequent proteasomal degradation. Elevated levels of cellular SUMOylation may lead to an 415 increase SUMOylation of PIAS1, prompting its localization to PML nuclear bodies and its 416 degradation by RNF4 in a ubiquitin-dependent manner. This feedback mechanism used to 417 regulate global SUMOylation may not be reserved solely for PIAS1. Indeed, other members of the 418 PIAS family, as well as NSE2 and TOPORS, have been found to be SUMOylated at several lysine 419 residues, while also being substrates of RNF4 ${ }^{54}$.

420 Notably, cytoskeletal proteins occupy a significant proportion of the identified PIAS1 421 substrates. Constituents of actin filaments, intermediate filaments and microtubules were all 422 found to be PIAS1 substrates. Interestingly, unlike UBC9 substrates that are typically SUMOylated 423 on consensus motifs ${ }^{6}$, the acceptor lysine residues found on these cytoskeletal proteins are highly 424 conserved but are located in non-consensus sequence motif. These observations suggest that 425 PIAS1 may act as an adaptor protein to change cytoskeletal protein turnover or dynamics by 426 facilitating their SUMOylation. We uncovered that SUMOylation on the tail domain of VIM 427 increases its solubility and promotes the uptake of ULF onto VIF in a phospho-dependent 428 mechanism. The dynamic VIF disassembly/reassembly that is promoted by VIM SUMOylation in 429 turn favors cell motility and invasion, which could lead to an increase in cancer cell aggressiveness. 
430 Although these findings could reveal the molecular mechanism of PIAS1 mediated VIM 431 SUMOylation and its involvement in cancer cell aggressiveness, additional evidence is required 432 to further understand the function of PIAS1-mediated SUMOylation on the other cytoskeletal 433 proteins and how these cytoskeletal proteins collaborate during cell migration.

434

435

436 


\section{METHODS}

438 Methods and any associated references are available in the online version of the paper.

439 Note: Supplementary information is available in the online version of the paper

\section{ACKNOWLEDGEMENTS}

442 This work was funded in part by the Natural Sciences and Engineering Research Council (NSERC)

443 (P.T., RGPIN-2018-04193) and the Canadian Institute for Health Research (CIHR) (G.E., PJT

444 148943, PJT 148560). C.L. was supported by a scholarship from Fonds de recherche du Québec-

445 Nature et technologies (FRQNT). The Institute for Research in Immunology and Cancer (IRIC)

446 receives infrastructure support from Genome Canada, the Canadian Center of Excellence in

447 Commercialization and Research, the Canadian Foundation for Innovation, and the Fonds de 448 recherche du Québec - Santé (FRQS).

\section{AUTHOR CONTRIBUTIONS}

451 C.L., F.P.M., C.P., C.M.P., T.N., L.E.A.D. performed experiments and analyzed data; C.L., F.P.M., 452 and P.T. wrote the manuscript; G.E. and P.T. developed the concept and managed the project.

\section{COMPETING FINANCIAL INTERESTS}

455 The authors declare no competing financial interests. 
Figure 1. Functional effects of PIAS1 overexpression on HeLa cells. (a) HeLa cells were transfected with Myc-PIAS1 or Empty vector (NC) for 48 hr. PIAS1 overexpression efficiency was determined by western blot. Actin was used as a loading control. (b) PIAS1 overexpression in HeLa cells significantly promotes cell growth. (c) PIAS1 overexpression increases cell migration as

462 determined by a wound-healing assay. (d) Quantification of the wound healing assay. (e)

463 Representative results from a Boyden chamber assay showing improved invasion capabilities of 464 PIAS1 overexpression in HeLa cells. (f) Quantification of the Boyden chamber assay. $\left({ }^{*} p<0.05\right.$, $465 \quad * * p<0.01$, Student's t-test).

466 Figure 2. Workflow for the identification of PIAS1 substrates. (a) HEK293 SUMO3m cells were 467 cultured in SILAC medium with reverse labeling in biological triplicates. For replicate 1, PIAS1 468 overexpression was performed in medium and heavy channels with Myc-PIAS1 vector, while the 469 cells cultured in light media were transfected with the pcDNA3.0 (Empty vector). For replicates 2 470 and 3 the empty vector was transduced in the medium and heavy labelled cells, respectively. (b)

471 Western blot showing the level of overexpression of PIAS1 in the transfected cells. Detection of 472 PIAS1 overexpression by both Anti-PIAS1 antibody and Anti-Myc antibody. $\beta$-Tubulin is used as 473 loading control. (c) SILAC labelled cells were lysed and combined in a 1:1:1 ratio based on protein 474 content. SUMOylated proteins were enriched from the cell extract on an IMAC column prior to 475 their tryptic digestion. After desalting and drying, peptides containing the SUMO3 remnant were 476 enriched using a custom anti-K-E-NQTGG antibody that was crosslinked on magnetic beads [4]. 477 Enriched peptides were further fractionated on SCX columns and injected on a Tribrid Fusion 478 mass spectrometer. Peptide identification and quantification were performed using MaxQuant.

479 Figure 3. Statistical analyses of mass spectrometry results and bioinformatics analyses of 480 identified PIAS1 substrates. (a) Volcano plots showing the global proteome changes in cells 481 overexpressing PIAS1 (Over.) vs. control cells (NC). Individual proteins are represented by points. 482 The area of the volcano plot where protein abundance changes are significantly regulated ( $p$ 483 value of $<0.05$ ) are shaded in pink. (b) Volcano plots showing the global SUMOylation changes in 484 cells overexpressing PIAS1 (Over.) vs. control cells (NC). Individual SUMOylation sites are 
represented by points. The area of the volcano plot where SUMO sites are significantly upregulated ( $p$-value of $<0.05$ ) is shaded in pink. (c) Summary of identified and quantified peptides and proteins in both the proteome and SUMOylome experiments. (d) Functional classification of PIAS1 substrates using PANTHER (Protein Analysis Through Evolutionary Relationships) (http://www.pantherdb.org). (e) GO term enrichment distribution of the identified PIAS1 substrates using DAVID 6.8 (https://david.ncifcrf.gov/).

Figure 4. Protein-Protein Interaction Network of PIAS1 substrates. STRING network of PIAS1

492 substrates and their interacting partners. Proteins are grouped according to their GO terms.

493 Figure 5. SUMOylation of PIAS1 promotes its PML localization. (a) Distribution of SUMO sites 494 identified on PIAS1. Three SUMO sites (K137, K237 and K315) were identified in the dataset. K137 495 and K238 are located in PINIT domain while K315 is located in SP-RING domain. (b) HEK293 496 SUMOm cells were co-transfected with PIAS1-GFP-WT or PIAS1-GFP-3xKR and 497 immunofluorescence was performed with anti-PML (scale bar, $10 \mu \mathrm{m}$ ). (c) Box plot graph showing 498 the ratio of PIAS1-PML co-localization relative to the total cell signal. $\left(^{* *} p<0.01\right.$, Student's t-test, $499 \mathrm{n}=10$ cells/condition)

500 Figure 6. SUMOylation of Vimentin (VIM) is required for proper cell growth, migration and 501 invasion in HeLa cells. (a) VIM sequence indicating SUMOylation sites at Lys 439 and Lys 445 502 localized in the tail domain, and highly conserved across six different species. (b) Western blot 503 analysis of HeLa cells transfected with an empty vector as a negative control, Wild-type vimentin 504 (Flag-VIM ${ }^{\mathrm{wt}}$ ), and Vimentin K439, 445R, double mutant (Flag-VIM ${ }^{\mathrm{mt}}$ ), showing the absence of 505 SUMOylation on the Flag-VIM ${ }^{\text {mt }}$ protein. (c) VIM ${ }^{m t}$ expression inhibits proper cell growth. (d) $506 \mathrm{VIM}^{\mathrm{mt}}$ expression inhibits proper cell migration as determined by a wound-healing assay. (e) 507 Quantification of the wound healing assay. (f) Effect of VIM ${ }^{\text {wt }}$ and VIM ${ }^{\text {mt }}$ overexpression on cell 508 invasion, as determined in a Boyden chamber assay. (g) VIM ${ }^{\mathrm{mt}}$ expression significantly inhibits 509 HeLa cell invasion. ( ${ }^{*} p<0.05,{ }^{*} p<0.01$, Student's t-test).

510 Figure 7. SUMOylation of Vimentin regulates its dynamic assembly. (a) HeLa cells were 511 transfected with an empty vector as a negative control, Flag-VIM ${ }^{\text {wt }}$ and Flag-VIM ${ }^{\mathrm{mt}}$ and separated 512 into RIPA soluble and insoluble fractions. VIM protein levels were examined by western blot. (b) 
513 MCF-7 cells were transfected with Emerald-wild-type vimentin (VIM ${ }^{\mathrm{wt}}$ ), and Emerald-vimentin

$514 \mathrm{~K} 439,445 \mathrm{R}$, double mutant ( $\mathrm{VIM}^{\mathrm{mt}}$ ), and the proportion of unit-length filament (ULF), vimentin

515 intermediate filament (VIF) and cytosolic vimentin between VIM ${ }^{\text {wt }}$ and VIM $^{\mathrm{mt}}$ was calculated

516 under microscope (scale bar, $10 \mu \mathrm{m}$ ).(c) Fluorescence Recovery After Photobleaching (FRAP)

517 assay of Emerald-wild-type vimentin ( $\mathrm{VIM}^{\mathrm{wt}}$ ), and Emerald-vimentin K439, 445R, double mutant

518 (VIM $\left.{ }^{\mathrm{mt}}\right)$ in MCF-7 cells. Line plot shows average fluorescence at each time point \pm s.e.m.

519 Differences between values for $\mathrm{VIM}^{\mathrm{wt}}$ and $\mathrm{VIM}^{\mathrm{mt}}$ were not statistically significant at all time points

520 by Student's t-test. (d) Model of the VIM dynamic assembly and disassembly. Vimentin is

521 maintained in equilibrium between Unit-length Filament (ULF) and soluble tetramers. (Subunit

522 exchange step). Vimentin filaments elongate by end-to-end annealing of ULF to form mature

523 vimentin intermediate filament (VIF; annealing step). The phosphorylation-dependent

524 shortening of VIF (severing step) involves phosphorylation on Ser 39 and Ser 56 of vimentin by

525 several kinases, including Akt1. The short filaments can reanneal with another ULF to form new

526 VIF (re-annealing step). However, the phosphorylated ULF are not amenable to the re-annealing

527 process. These phosphorylated ULF products are subject to PIAS1-mediated SUMOylation,

528 stimulating the dephosphorylation of the phosphorylated ULF, and subsequently reenter to

529 either subunit exchange process or VIF maturation process.

530

531 


\section{Figure 1:}

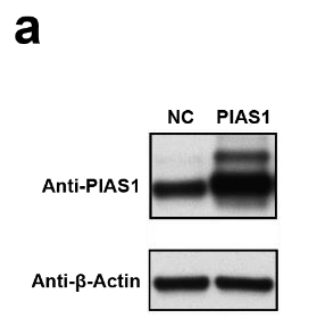

b
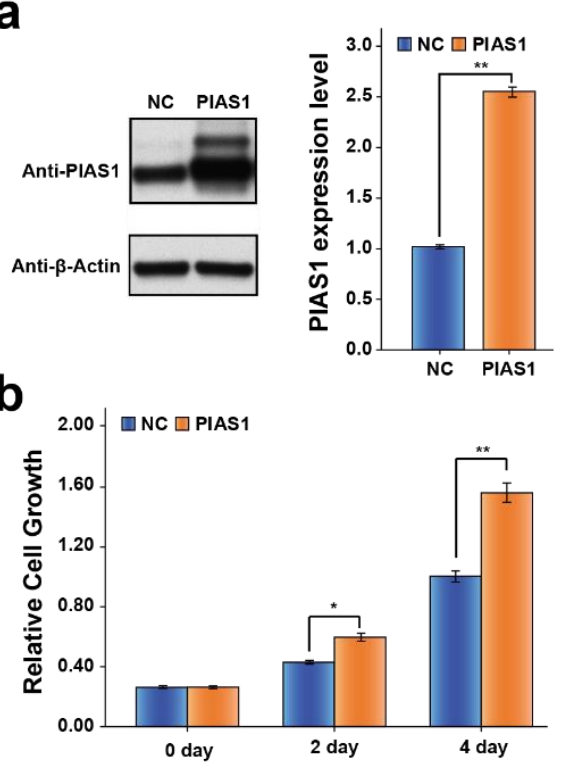

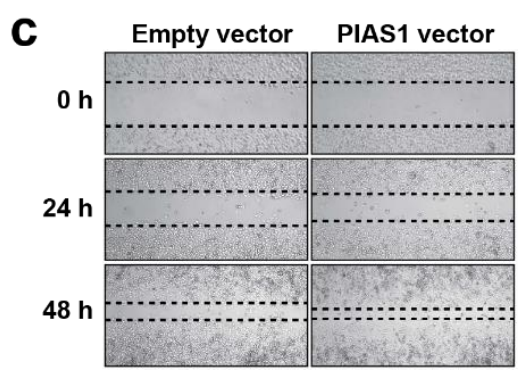

d

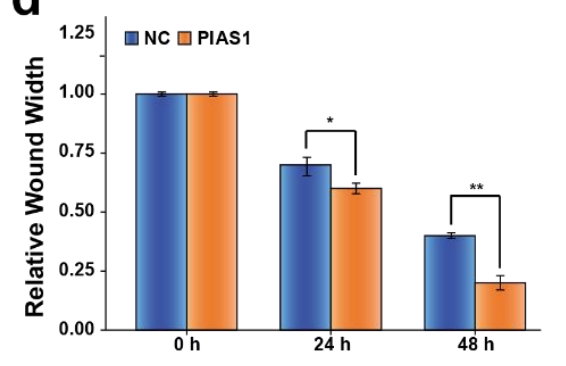

e

NC

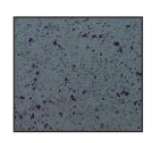

PIAS1

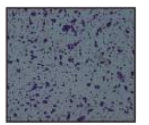

f

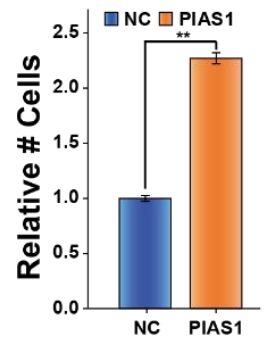




\section{Figure 2:}

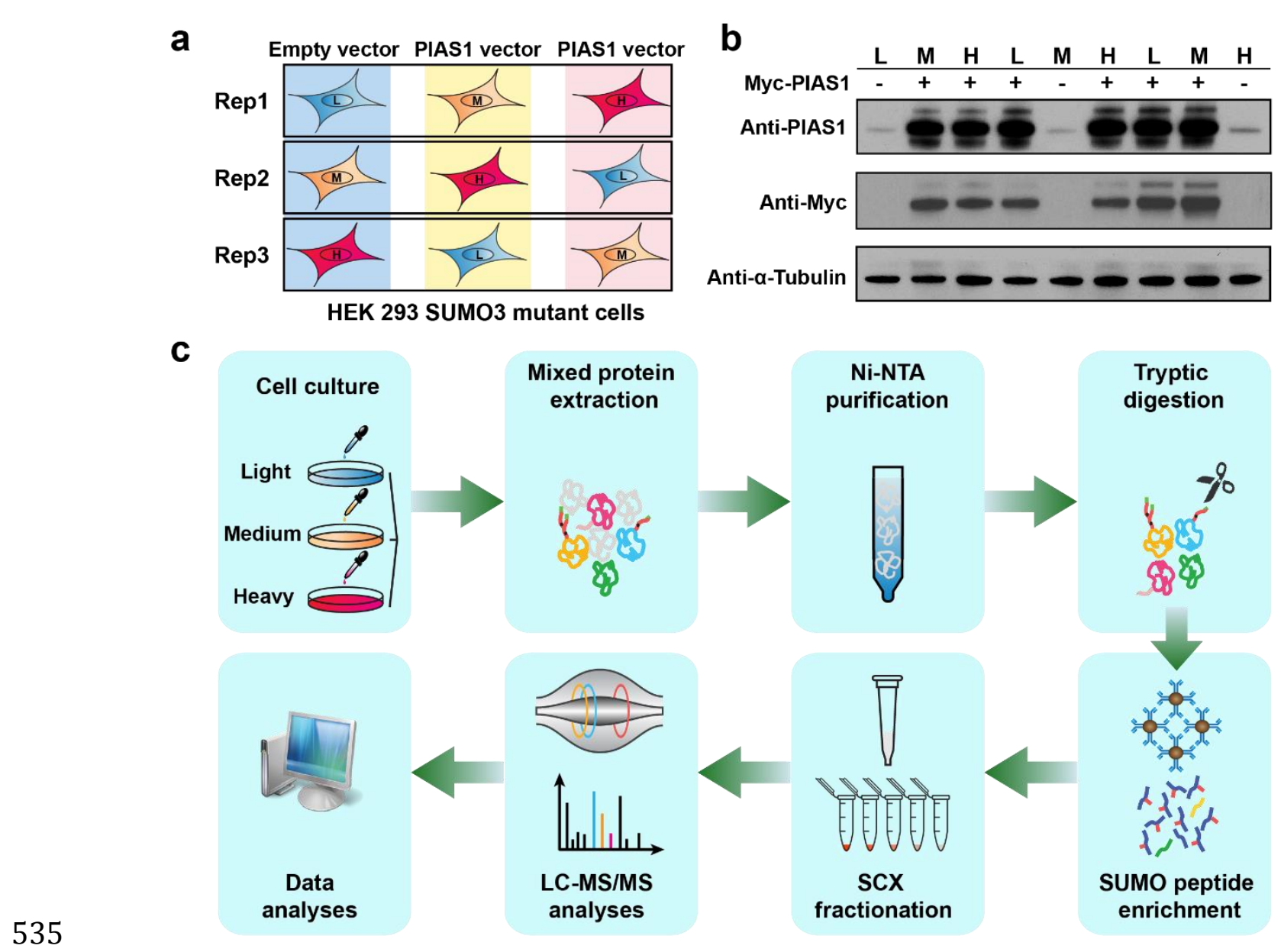




\section{Figure 3:}

a

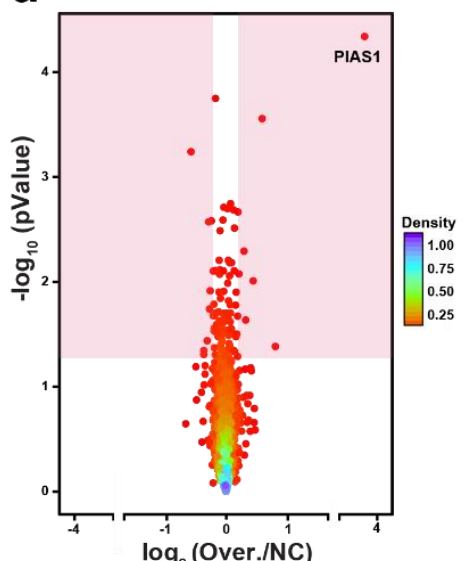

b

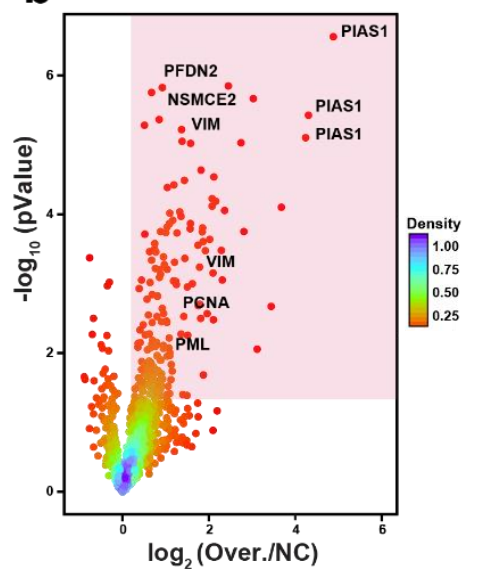

\section{C}

\begin{tabular}{|l|l|}
\hline Proteome result & Number \\
\hline Identified peptides & 20764 \\
\hline Identified proteins & 3033 \\
\hline Quantified peptides & 12080 \\
\hline Quantified proteins & 1756 \\
\hline SUMO proteome result & Number \\
\hline Identified SUMO peptides & 1435 \\
\hline Identified SUMO proteins & 705 \\
\hline Quantified SUMO peptides & 983 \\
\hline Quantified SUMO proteins & 544 \\
\hline Regulated SUMO sites & 204 \\
\hline Putative substrates & 123 \\
\hline
\end{tabular}

d

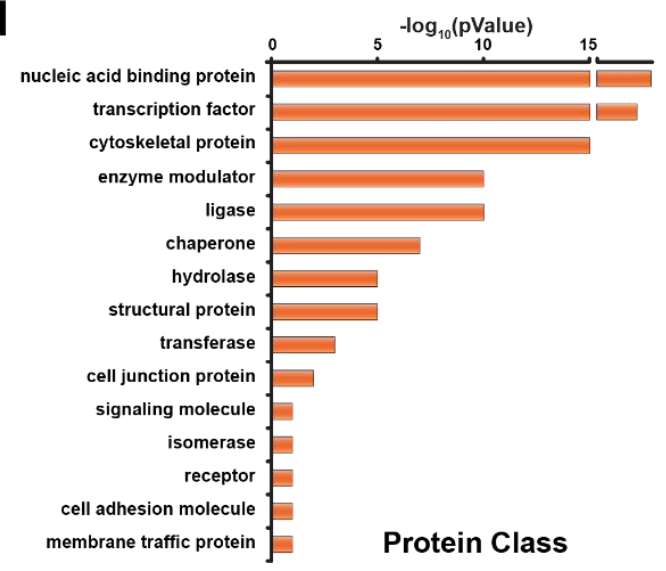

e

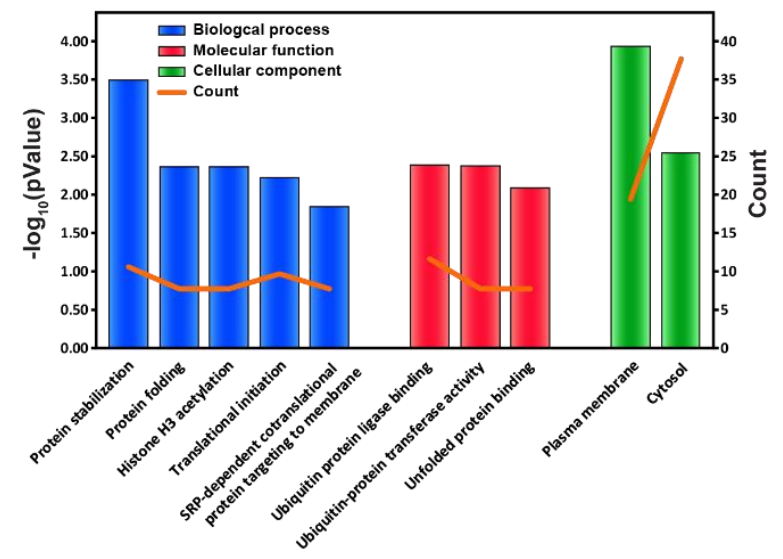




\section{Figure 4:}

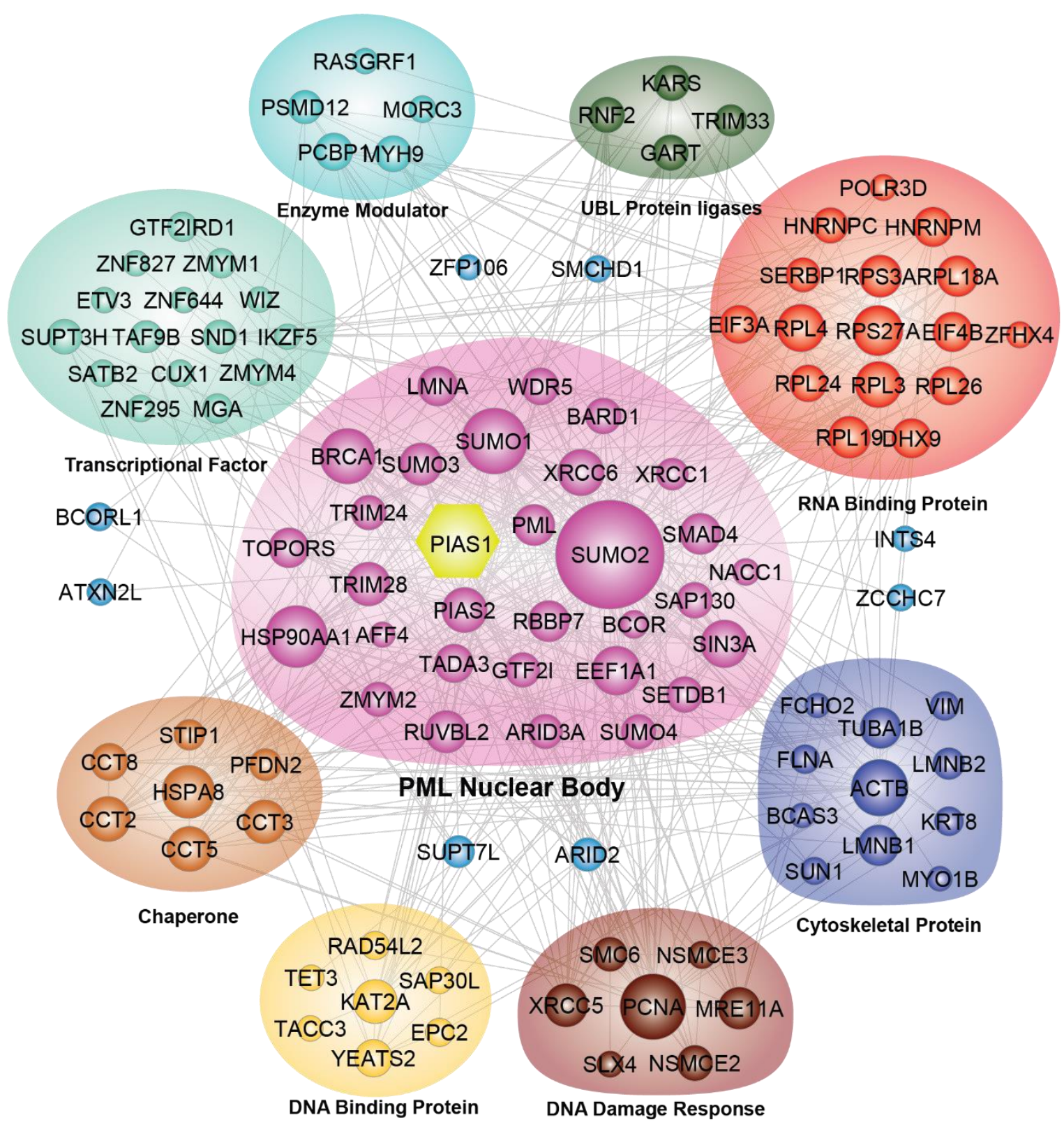




\section{Figure 5:}

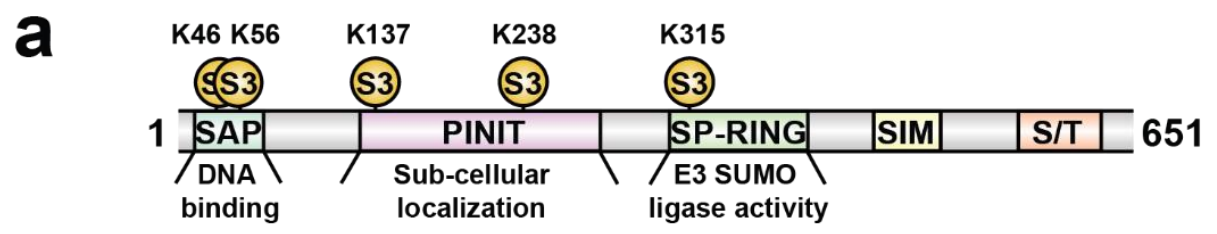

b

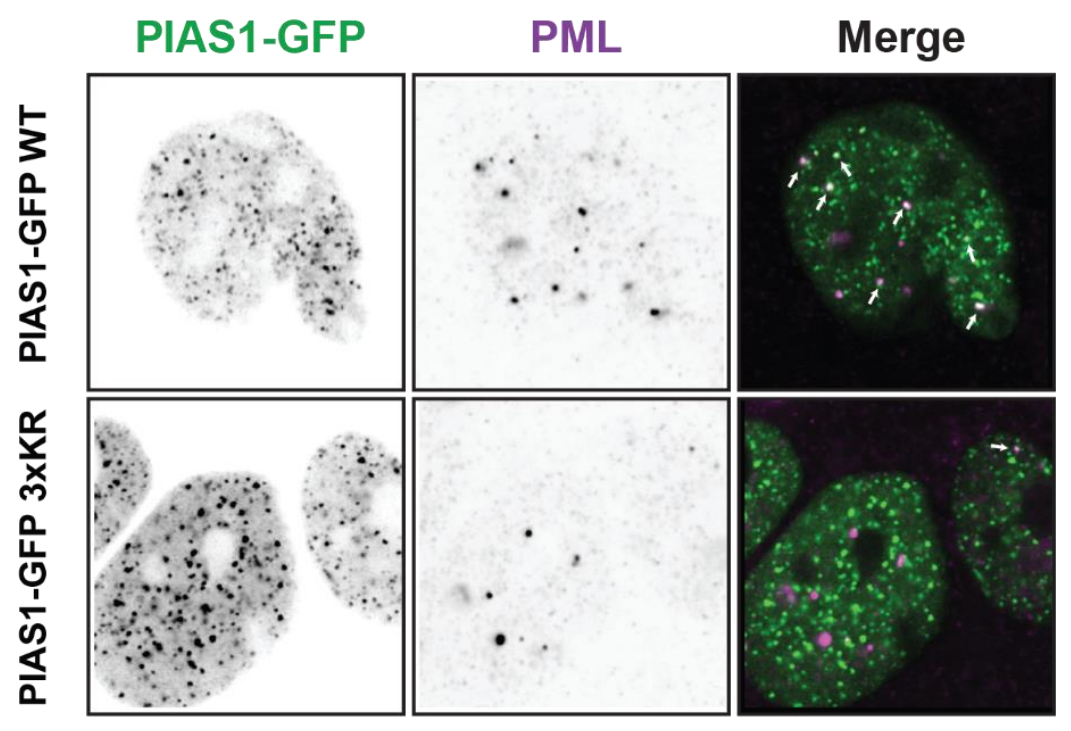

C

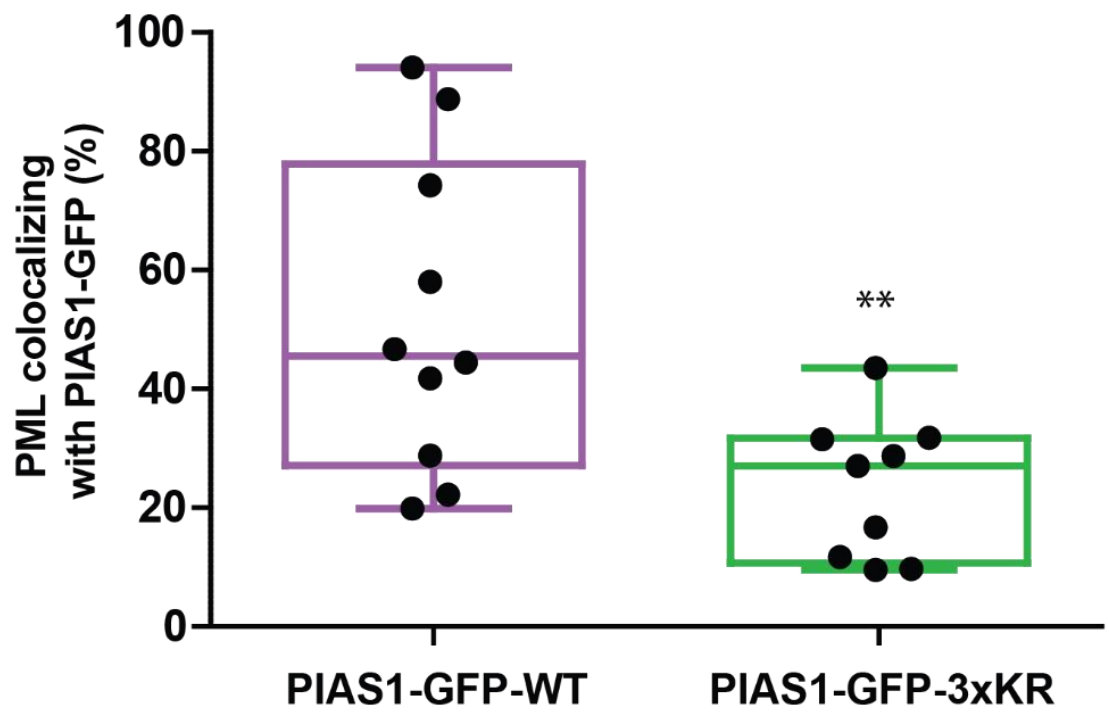




\section{Figure 6:}

a

Vimentin

K439 K445

1

Head

' Co

\section{$\mathrm{VIM}^{\mathrm{mt}}$ :}

H. sapiens:

M. musculus:

B. taurus:

G. gallus:

Vimentin

(\$3) (\$3)

X. tropicalis:

D. rerio:

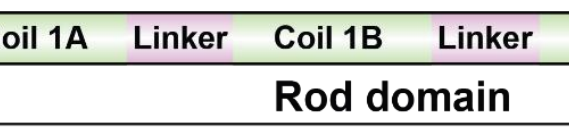

428 LDSLPLVDTHSKRTLIIKTVETRDGQVINETSQHHDDLE 466

428 LESLPLVDTHSKRTLLIKTVETRDGQVINETSQHHDDLE 466

428 LDSLPLVDTHSKRTLLIKTVETRDGQVINETSQHHDDLE 466

412 IESQPIVDTHSKRTLLIKTVETRDGQVINETSQHHDDLE 460

413 LDSHPV-ESHSKRTLLIKTVETRDGQVINESSQHHDDLE 461

417 KETRPLIDNLSKK-VVIKTIETRDGHIINESTQN-EDLE 455

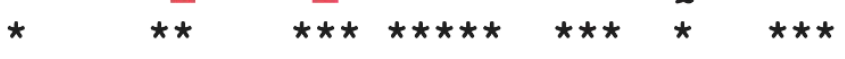

428 LDSLPLVDTHSRRTLIIRTVETRDGQVINETSQHHDDLE 466

b

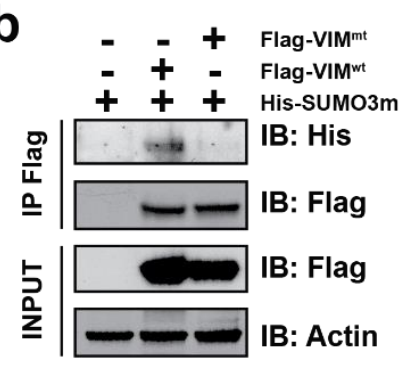

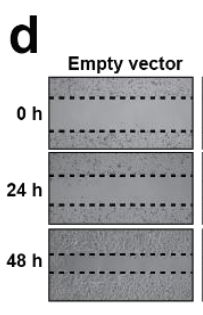

C
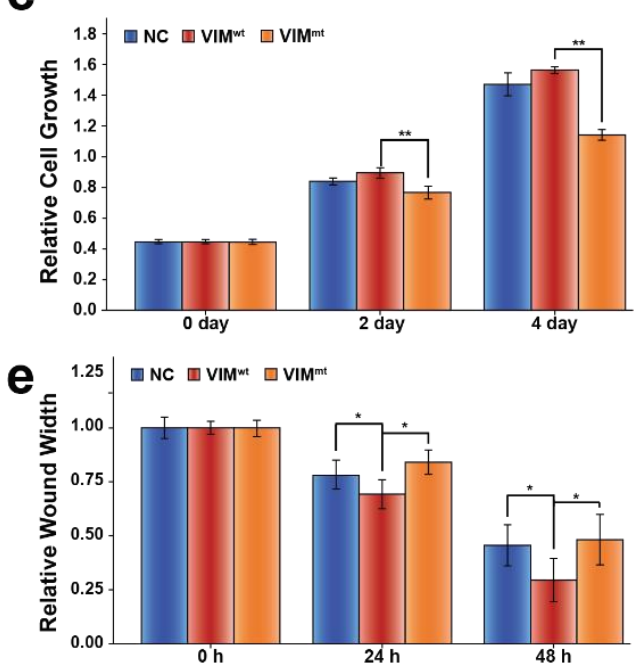

f
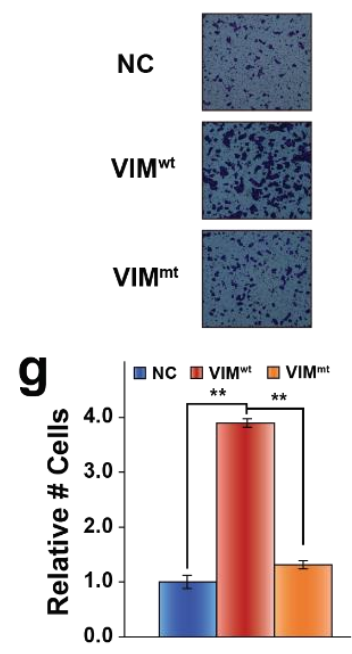


\section{Figure 7:}

a
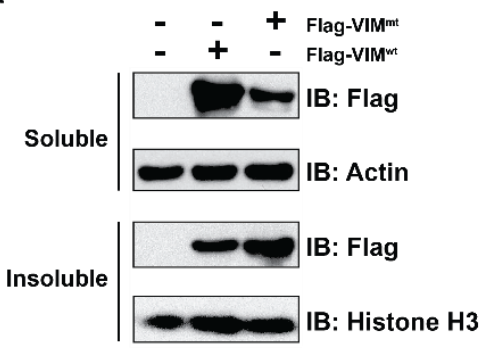

d

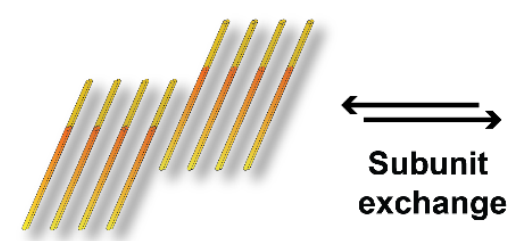

Soluble tetramers
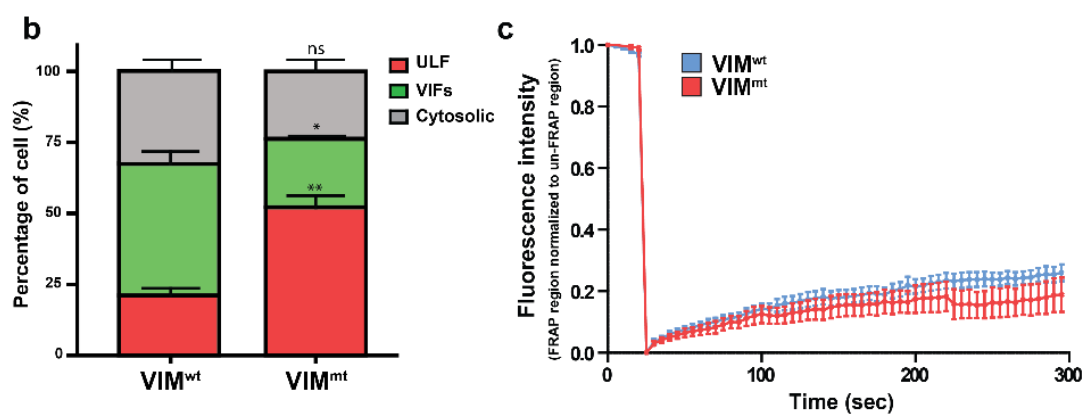

Vimentin intermediate

filaments (VIFs)

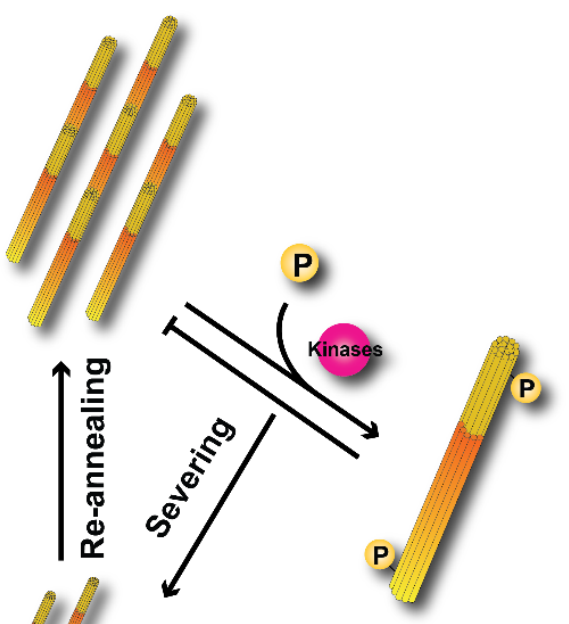

Phosphorylated ULF

\section{Unit-length filament (ULF)}
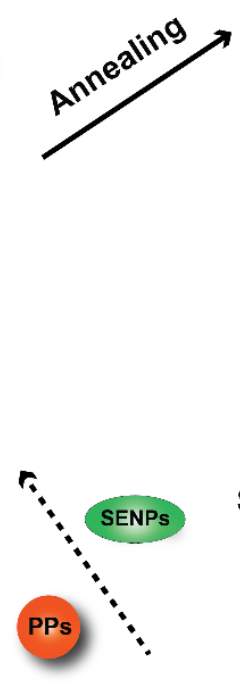

Short filaments

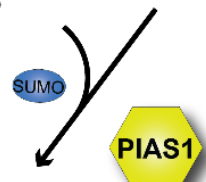

SUMOylated \& Phosphorylated ULF 


\section{METHODS}

548 Methods and any associated references are available in the online version of the paper.

549 Note: Supplementary information is available in the online version of the paper

\section{Online Methods}

\section{Cell Culture and Transfection}

554 Human cervical cancer cell line (HeLa) and HEK293 stably expressing the 6xHis-SUMO3-Q87R/Q88N 555 mutant (HEK293-SUMO3m) ${ }^{27}$ were cultured in Dulbecco's modified Eagle's medium (HyClone) 556 supplemented with $10 \%$ fetal bovine serum (Wisent), 1\% L-glutamine (Thermo Fisher Scientific), 1\% 557 penicillin/streptomycin (Invitrogen) in $5 \% \mathrm{CO} 2$ at $37^{\circ} \mathrm{C}$. The mammalian expression vector for Myc-PIAS1 558 and 6xHis-SUMO3 were constructed by inserting the full-length cDNAs into pcDNA3.0-Myc and pcDNA3.0-

$5596 x$ His vectors, respectively. The mammalian expression vector for PIAS1-GFP-WT was constructed by 560 cloning full-length PIAS1 cDNA into pcDNA3.1-c-GFP10. The PIAS1-GFP-3xKR plasmid was generated by 561 site-directed mutagenesis using the GENEART Site-Directed Mutagenesis System according to the 562 instructions of the manufacturer (InvitrogenTM). The mammalian expression vectors pReceiver-M11 563 (Flag-NSMCE2, Flag-PFDN2 and Flag-VIM) and the bacterial expression vectors pReceiver-B11 (His564 NSMCE2 and His-PFDN2) were purchased from Genecopoeia, Inc. (Rockville, MD).

565 For transient transfection, HeLa cells or HEK293-SUMO3m cells were transfected with $1 \mu \mathrm{g}$ plasmid per 566 million cells using JetPrime Reagent (Polyplus-transfection) according to the manufacturer's protocol. 567 Cells were collected $48 \mathrm{~h}$ after transfection and protein overexpression was confirmed by western blot.

\section{Cell Proliferation Assay}

569 The cell proliferation assay was carried out using WST-1 Cell Proliferation Assay Kit (Roche). Transfected 570 cells were seeded into 96-well plates at a density of $1 \times 10^{3}$ cells/well. After 0,2 and 4 days of incubation, 571 WST-1 reagents were added to each well, and cells were further incubated at $37{ }^{\circ} \mathrm{C}$ for $1 \mathrm{~h}$. Each 572 measurement was performed in triplicate, and the experiments were repeated three times. The relative 573 numbers of viable cells were estimated using the absorbance optical density (OD) at $450 \mathrm{~nm}$. 


\section{Migration Assays}

576 Cell migration was assessed by using a wound-healing assay. Transfected cells were seeded as a single 577 monolayer into 12 -well plates at $80 \%-90 \%$ confluence and then scratched using a $200-\mu$ l pipette tip. After 578 the scratch, the cells were washed twice with PBS to remove the cell debris and grown in DMEM 579 containing $1 \%$ FBS. Phase-contrast microscopy images of the wound area were taken at time $0 \mathrm{~h}, 24 \mathrm{~h}$ and $58048 \mathrm{~h}$, wound healing was estimated by measuring the relative width.

\section{In vitro Transwell Invasion Assays}

582 Invasive ability of transfected HeLa cells was assessed using BioCoat Matrigel Invasion Chamber (Corning) 583 which consists of a 24-well Falcon TC Companion Plate and $8 \mu \mathrm{m}$ pore size PET membrane with a thin layer 584 of MATRIGEL Basement Membrane Matrix. Chambers were rehydrated by adding $0.5 \mathrm{ml}$ DMEM 585 containing $10 \%$ FBS to the interior of the inserts and bottoms of wells. The chambers were incubated in a 586 humidified tissue culture incubator at $37^{\circ} \mathrm{C}$ for $2 \mathrm{~h}$. After rehydration, the medium was carefully removed 587 without disturbing the layer of Matrigel. Transfected HeLa cells were seeded at $2.5 \times 10^{4}$ cells in $0.5 \mathrm{ml}$ of 588 serum-free DMEM to the interior of the inserts. DMEM containing 10\% FBS was added to the bottoms of 589 wells. Following a $48 \mathrm{~h}$ incubation at $37^{\circ} \mathrm{C}$, adherent cells on the upper layer of the insert were removed 590 by gentle scraping with a cotton tip applicator. Cells that had invaded the underside of the inserts were 591 fixed with $100 \%$ methanol for 10 minutes at $-20^{\circ} \mathrm{C}$ and stained with $0.5 \%$ crystal violet dye (EMD Millipore) 592 for $10 \mathrm{~min}$ at room temperature. The permeated cells were imaged under a Phase-contrast microscope.

593 Then cells were incubated with $10 \%$ acetic acid to elute the dye and the relative cell numbers were 594 estimated using the absorbance optical density (OD) at $590 \mathrm{~nm}$.

\section{SILAC Labeling and Protein Extraction}

596 HEK293-SUMO3m cells were grown in DMEM (Thermo Fisher Scientific) containing light (OLys, OArg), 597 medium (4Lys, 6Arg) or heavy (8Lys, 10Arg) isotopic forms of lysine and arginine (Silantes) for at least 6 598 passages to ensure full labeling. For each triple SILAC experiment, the control channel was transfected 599 with an empty-pcDNA3.0-Myc vector while the other two channels were transfected with the Myc-PIAS1 600 plasmid. After $48 \mathrm{~h}$ transfection, an equal amount of cells from each SILAC channel were combined and 601 washed twice with ice-cold PBS, lysed in NiNTA denaturing incubation buffer (6 M Guanidinium HCl, 100 $602 \mathrm{mM} \mathrm{NaH} \mathrm{PO}_{4}, 20 \mathrm{mM}$ 2-Chloroacetamide, $5 \mathrm{mM}$ 2-Mercaptoethanol, $10 \mathrm{mM} \mathrm{Tris-HCl} \mathrm{pH=8)} \mathrm{and}$ 603 sonicated. Protein concentration was determined using micro Bradford assay (Bio-Rad). 


\section{Protein Purification, Digestion and Desalting}

605 For each replicate, $16 \mathrm{mg}$ of total cell extract (TCE) were incubated with $320 \mu \mathrm{L}$ of NiNTA beads (Qiagen)

606 at $4{ }^{\circ} \mathrm{C}$. After $16 \mathrm{~h}$ incubation, NiNTA beads were washed once with $10 \mathrm{~mL}$ of NiNTA denaturing incubation

607 buffer, 5 times with $10 \mathrm{~mL}$ of NiNTA denaturing washing buffer (8 M urea, $100 \mathrm{mM} \mathrm{NaH}{ }_{2} \mathrm{PO}_{4}, 20 \mathrm{mM}$

608 imidazole, $5 \mathrm{mM}$ 2-Mercaptoethanol, $20 \mathrm{mM}$ Chloroacetamide, $10 \mathrm{mM}$ Tris- $\mathrm{HCl} \mathrm{pH=6.3)} \mathrm{and} \mathrm{twice} \mathrm{with}$

$60910 \mathrm{~mL}$ of $100 \mathrm{mM}$ ammonium bicarbonate. Protein concentration was determined using micro Bradford

610 assay (Bio-Rad). Protein digestion on beads was carried out using a ratio 1:50 sequencing grade modified

611 trypsin (Promega): protein extract in $100 \mathrm{mM}$ ammonium bicarbonate at $37^{\circ} \mathrm{C}$ overnight. To quench the

612 reaction, $0.1 \%$ trifluoroacetic acid (TFA) was added. The solution was desalted on hydrophilic-lipophilic

613 balance (HLB) cartridges (1cc, $30 \mathrm{mg}$ )(Waters) and eluted in LoBind tubes (Eppendorf) before being dried

614 down by Speed Vac.

\section{SUMO Peptide Enrichment}

616 PureProteome protein A/G magnetic beads (Millipore) were equilibrated with anti-K(NQTGG) antibody at 617 a ratio of $1: 2(\mathrm{v} / \mathrm{w})$ for $1 \mathrm{~h}$ at $4{ }^{\circ} \mathrm{C}$ in PBS. Saturated beads were washed 3 times with $200 \mathrm{mM}$ 618 triethanolamine $\mathrm{pH}=8.3$. For crosslinking, $10 \mu \mathrm{l}$ of $5 \mathrm{mM}$ DMP in $200 \mathrm{mM}$ triethanolamine $\mathrm{pH}=8.3$ was 619 added per $\mu$ of slurry and incubated for $1 \mathrm{~h}$ at room temperature. The reaction was quenched for 30 620 minutes by adding $1 \mathrm{M}$ Tris- $\mathrm{HCl} \mathrm{pH=8}$ to a final concentration $5 \mathrm{mM}$. Cross-linked beads were washed 3 621 times with ice-cold PBS and once with PBS containing 50\% glycerol. The tryptic digests were resuspended 622 in 500 ul PBS containing 50\% glycerol and supplemented with cross-linked anti-K-(NQTGG) at a ratio of $6231: 2(\mathrm{w} / \mathrm{w})$. After $1 \mathrm{~h}$ incubation at $4{ }^{\circ} \mathrm{C}$, anti-K-(NQTGG) antibody bound beads were washed three times 624 with $1 \mathrm{ml}$ of $1 \times$ PBS, twice with $1 \mathrm{ml}$ of $0.1 \times$ PBS and once with ddH2O. SUMO peptides were eluted three 625 times with $200 \mu$ l of $0.2 \%$ formic acid in water and dried down by Speed Vac.

\section{SCX Fractionation}

627 Enriched SUMO peptides were reconstituted in water containing 15\% acetonitrile and 0.2\% formic acid 628 and loaded on conditioned strong cation exchange (SCX) StageTips (Thermo Fisher Scientific). Peptides 629 were eluted with ammonium formate pulses at 50, 75, 100, 300, 600 and 1,500 mM in 15\% acetonitrile, $630 \mathrm{pH}=3$. Eluted fractions were dried down by Speed Vac and stored at $-80^{\circ} \mathrm{C}$ for MS analysis. 


\section{Mass Spectrometry Analysis}

632 Peptides were reconstituted in water containing $0.2 \%$ formic acid and analyzed by nanoflow-LC-MS/MS 633 using an Orbitrap Fusion Mass spectrometer (Thermo Fisher Scientific) coupled to a Proxeon Easy-nLC 634 1000. Samples were injected on a $300 \mu \mathrm{m} \mathrm{ID} \times 5 \mathrm{~mm}$ trap and separated on a $150 \mu \mathrm{m} \times 20 \mathrm{~cm}$ nano-LC 635 column (Jupiter C18, $3 \mu \mathrm{m}, 300 \mathrm{~A}$, Phenomenex). The separation was performed on a linear gradient from 6367 to $30 \%$ acetonitrile, $0.2 \%$ formic acid over 105 minutes at $600 \mathrm{nl} / \mathrm{min}$. Full MS scans were acquired from $637 \mathrm{~m} / \mathrm{z} 350$ to $\mathrm{m} / \mathrm{z} 1,500$ at resolution 120,000 at $\mathrm{m} / \mathrm{z} 200$, with a target AGC of $1 \mathrm{E} 6$ and a maximum injection 638 time of $200 \mathrm{~ms}$. MS/MS scans were acquired in HCD mode with a normalized collision energy of 25 and 639 resolution of 30,000 using a Top $3 \mathrm{~s}$ method, with a target AGC of $5 E 3$ and a maximum injection time of $6403,000 \mathrm{~ms}$. The MS/MS triggering threshold was set at $1 \mathrm{E} 5$ and the dynamic exclusion of previously 641 acquired precursor was enabled for $20 \mathrm{~s}$ within a mass range of $\pm 0.8 \mathrm{Da}$.

\section{Data Processing}

643 MS data were analyzed using MaxQuant (version 1.5.3.8) ${ }^{55,56}$. MS/MS spectra were searched against 644 UniProt/SwissProt database (http://www.uniprot.org/) including Isoforms (released on 10 March 2015). 645 The maximum missed cleavage sites for trypsin was set to 2. Carbamidomethylation (C) was set as a fixed 646 modification and acetylation (Protein N term), oxidation (M), deamination (NQ) and NQTGG (K) were set 647 as variable modifications. The option match between runs was enabled to correlate identification and 648 quantitation results across different runs. The false discovery rate for peptide, protein, and site 649 identification was set to $1 \%$. SUMO sites with a localization probability of $>0.75$ were retained. The mass 650 spectrometry proteomics data have been deposited to the ProteomeXchange Consortium 651 (http://proteomecentral.proteomexchange.org) via the PRIDE partner repository with the dataset 652 identifier <PXD011932>. Reviewer account details: Username: reviewer91455@ebi.ac.uk, Password: 653 ScCPBsuC

\section{Bioinformatics Analysis}

655 Classification of identified PIAS1 substrates was performed using PANTHER (Protein Analysis Through 656 Evolutionary Relationships) (http://www.pantherdb.org), which classifies genes and proteins by their 657 functions ${ }^{57,58}$. The identified PIAS1 substrates were grouped into the biological process, molecular 658 function and cellular component classes against the background of quantified SUMOylome using DAVID 659 Bioinformatics Resources $6.7^{59}$. The aligned peptide sequences with \pm 6 amino acids sounding the modified 660 Iysine residue obtained in Andromeda were submitted to IceLogo ${ }^{60}$. For peptide sequences corresponding 
661 to multiple proteins, only the leading sequence was submitted. The secondary structures surrounding the

662 PIAS1-regulated SUMO sites were investigated using NetSurfP-1.1 (Petersen et al. 2009) ${ }^{61}$. INTERPRO

663 Protein Domains Analysis and Protein-Protein Interaction (PPI) network of identified PIAS1 substrates

664 were built by searching against the STRING (Search Tool for the Retrieval of Interacting Genes/Proteins)

665 database version 9.162,63. All predictions were based on experimental evidence with the minimal

666 confidence score of 0.4 , which is considered as the highest confidence filter in STRING. PPI networks were

667 then visualized by Cytoscape v3.5.1 $1^{64,65}$.

\section{Recombinant Protein Purification and in vitro SUMO Assay}

669 The bacterial expression vectors pReceiver-B11 for His-NSMCE2 and His-PFDN2 recombinant protein 670 expression were purchased from Genecopoeia, Inc (Rockville, MD). pReceiver-B11 was individually 671 transformed into ArcticExpress Competent Cells. Transformed bacteria were cultured in LB medium until 672 an OD600 of 0.5. Isopropyl $\beta$-D-1-thiogalactopyranoside (IPTG) (Bioshop) was added to the culture at a 673 final concentration of $1 \mathrm{mM}$ and the desired expression of the protein was inducted for $24 \mathrm{~h}$ at $13^{\circ} \mathrm{C}$. 674 Harvested bacterial pellets were lysed and sonicated in a solution containing $500 \mathrm{mM} \mathrm{NaCl}, 5 \mathrm{mM}$ 675 imidazole, $5 \mathrm{mM} \beta$-Mercaptoethanol, $50 \mathrm{mM}$ Tris- $\mathrm{HCl} \mathrm{pH=7.5}$. His-tagged proteins were purified on NiNTA 676 beads (Qiagen). Purified proteins were eluted with $500 \mathrm{mM} \mathrm{NaCl}, 250 \mathrm{mM}$ imidazole, $5 \mathrm{mM} \beta$ 677 mercaptoethanol, $50 \mathrm{mM}$ Tris- $\mathrm{HCl} \mathrm{pH=7.5}$ and concentrated on $3 \mathrm{kDa}$ Ultra centrifugal filters (Amicon).

678 Recombinant human PCNA protein was a generous gift from Dr. Alain Verreault (University of Montreal, 679 Canada). The in vitro SUMO assay was carried out in a buffer containing $5 \mathrm{mM} \mathrm{MgCl}_{2}, 5 \mathrm{mM} \mathrm{ATP}, 50 \mathrm{mM}$

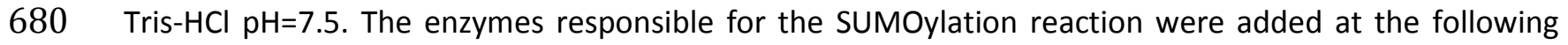
681 concentrations: $0.1 \mu \mathrm{M}$ SAE1/2, $1 \mu \mathrm{M}$ Ubc9, $10 \mu \mathrm{M}$ SUMO-3 and $60 \mathrm{nM}$ PIAS1. The reactions were 682 incubated at $37^{\circ} \mathrm{C}$ for $4 \mathrm{~h}$ and analyzed by western blot using an anti-His antibody.

\section{In vivo SUMO Assay and Immunoprecipitation}

684 HEK293-SUMO3m cells were co-transfected with Myc-PIAS1, Flag-NSMCE2, Flag-PFDN2 or Flag-VIM. At $68548 \mathrm{~h}$ post-transfection, cells were harvested and lysed with $1 \mathrm{ml}$ of Triton lysis buffer (150 mM NaCl, 0.2\% 686 Triton X-100, $1 \mathrm{mM}$ EDTA, 10\% Glycerol, $50 \mathrm{mM}$ Tris-HCl pH 7.5) supplemented with protease inhibitor 687 cocktail (Sigma Aldrich) at $4{ }^{\circ} \mathrm{C}$ for $15 \mathrm{~min}$ with gentle rocking. TCE were incubated with anti-Flag M2 688 Affinity Agarose Gel (Sigma Aldrich) with gentle rocking at $4{ }^{\circ} \mathrm{C}$ for overnight. Immunoprecipitates were 689 then washed three times with cold Triton lysis buffer and were analyzed by western blot using an anti-His 690 antibody (Sigma Aldrich). 
693 TCE prepared in Triton lysis buffer were diluted in Laemmli buffer (10\% (w/v) glycerol, 2\% SDS, 10\% (v/v)

694 2-mercaptoethanol and $62.5 \mathrm{mM}$ Tris- $\mathrm{HCl}, \mathrm{pH}=6.8$ ), boiled for $10 \mathrm{~min}$ and separated on a 4-12\% SDS695 PAGE (Bio-rad) followed by transfer onto nitrocellulose membranes. Before blocking the membrane for 1 $696 \mathrm{~h}$ with 5\% non-fat milk in TBST (Tris-buffered saline with Tween 20), membranes were briefly stained with $6970.1 \%$ Ponceau-S in 5\% acetic acid to represent total protein content. Membranes were subsequently 698 incubated overnight with a 1:1000 dilution of antibodies at $4{ }^{\circ} \mathrm{C}$. Membranes were then incubated with 699 peroxidase-conjugated anti-mouse or anti-rabbit IgG (Cell Signaling Technology) for $1 \mathrm{~h}$ at room 700 temperature at a 1:5000 dilution. Membranes were washed three times with TBST for 10 minutes each 701 and revealed using ECL (GE Healthcare) as per the manufacturer's instructions. Chemiluminescence was 702 captured on Blue Ray film (VWR). The following antibodies were used for western blot analyses: rabbit 703 anti-Flag polyclonal (Sigma Aldrich), rabbit anti-PIAS1 polyclonal (Cell Signaling Technology), rabbit anti704 Myc polyclonal (Cell Signaling Technology), rabbit anti-His (Sigma Aldrich), rabbit anti-Tubulin polyclonal 705 antibody (Cell Signaling Technology).

\section{Fluorescence imaging and co-localization analysis}

707 HEK-SUMO3m cells were plated on 12-mm-diameter coverslips until they reached the desired density 708 level and then transfected with the desired plasmids for $48 \mathrm{~h}$. Cells were fixed in $4 \%$ paraformaldehyde 709 (in PBS) for $15 \mathrm{~min}$, followed by a 2-min permeabilization with 0.1\% Triton X-100 (in PBS) and saturation 710 with 2\% BSA (in PBS) for 15 min. Cells were incubated with the primary antibodies for $1 \mathrm{~h}$ at $37^{\circ} \mathrm{C}$, rinsed 711 and incubated with secondary antibodies conjugated to Alexa Fluor (Cell Signaling Technology) and DAPI 712 (Sigma Aldrich) for $1 \mathrm{~h}$ at room temperature. Both primary and secondary antibodies are diluted in PBS / 713 BSA 2\%. To reach sub-diffraction resolution, images from fixed samples were acquired with the Airyscan 714 detector of a Zeiss LSM880 confocal equipped with a 63X/1.43 Plan Apochromat oil immersion objective. 715 We automatically detected and assessed the number of PIAS1 and PML positive structures by using the 716 "particle analysis" tool from ImageJ. We then calculated the ratio manually.

\section{In-gel Digestion and LC-MS/MS Identification}

718 Cell pellets were resuspended in 5 pellet volumes of ice cold RIPA buffer. The lysate was spun at $13000 \mathrm{~g}$ 719 for 10 minutes to separate the extract into RIPA soluble and insoluble fractions. The soluble and insoluble 
720 VIM samples were separated on a 4-12\% SDS-PAGE (Bio-rad), and the proteins were visualized by

721 coomassie staining. The gel lane around vimentin corresponding position ( $57 \mathrm{kDa}$ ) was cut and then diced

722 into $\sim 1 \mathrm{~mm}^{3}$ cubes. During the process of in-gel digestion, the gel pieces were first destained completely

723 using destaining solution $\left(50 \% \mathrm{H}_{2} \mathrm{O}, 40 \%\right.$ methanol, and $10 \%$ acetic acid). Then the gel pieces were

724 dehydrated by washing several times in 50\% acetonitrile (ACN) until the gel pieces shriveled and looked

725 completely white. The proteins were reduced in $10 \mathrm{mM}$ DTT at $56^{\circ} \mathrm{C}$ for $30 \mathrm{~min}$, alkylated in $55 \mathrm{mM}$

726 iodoacetamide at room temperature (RT) in the dark for $30 \mathrm{~min}$, and digested overnight with $300 \mathrm{ng}$ of

727 sequencing grade modified trypsin in $50 \mathrm{mM}$ ammonium bicarbonate. The supernatants were transferred

728 into Eppendorf tubes, and the gel pieces were sonicated twice in extraction buffer (67\% ACN and $2.5 \%$

729 trifluoroacetic acid). Finally, the peptide extraction and the initial digest solution supernatant were

730 combined and then dried using a Speed Vac. Peptides were reconstituted in water containing $0.2 \%$ formic

731 acid and analyzed by nanoflow-LC-MS/MS using an Orbitrap Q Exactive HF Mass spectrometer (Thermo

732 Fisher Scientific) as described previously ${ }^{66}$.

\section{Fluorescence Recovery After Photobleaching (FRAP) assay}

734 The FRAP assays were conducted on a LSM 880 confocal microscope equipped with a thermostatized 735 chamber at $37^{\circ} \mathrm{C}$. The Vimentin Emerald expressing cells were detected using a GaAsp detector. Bleaching 736 was done by combining "Time", "Bleach" and "Region" modes on Zen software from Zeiss. Briefly, 5 pre737 bleach images were taken every 5 seconds, after which five pulses of a $488 \mathrm{~nm}$ laser were applied to 738 bleach an area of $25 \times 2 \mu \mathrm{m}$. Post-bleach images were acquired every 5 seconds for 5 minutes. For

739 fluorescence recovery analysis, the intensity in the bleached region was measured varying time points 740 with "Frap profiler" plugin in ImageJ software. Bleach data were normalized to unbleached regions for all 741 the time points and expressed in arbitrary units in the recovery graphs.

\section{Quantification of Vimentin organization.}

743 Fixed images of cells expressing Emerald-VIM ${ }^{\text {wt }}$ or Emerald-VIM ${ }^{\text {mt }}$ were taken using a LSM 880 confocal 744 microscope. The "title" mode in the Zen software from Zeiss was used to cover a large area of cells $745(2,13 \mathrm{~mm} \times 2,13 \mathrm{~mm})$. To avoid localization and conformation artefacts due to expression levels, only the 746 cells expressing Emerald-Vimentin at an intermediate level were evaluated using the threshold module in 747 ImageJ. The same manual threshold was used for all conditions. Cells were tabulated using the "Cell 748 counter" plugin in ImageJ into 4 categories: ULF, VIFs, cytosolic and total cells. Categories specification 
749 were performed manually and subjectively. A representative image of each category is shown in Figure 750 S9. More than 250 cells were counted for each experiments ( $N=3, n$ total cells $=750$ ).

751

\section{Statistical Analysis}

753 Statistical analysis was carried out to assess differences between experimental groups. Statistical

754 significance was analyzed by the Student's t-tests. $p<0.05$ was considered to be statistically significant.

755 One asterisk and two asterisks indicate $p<0.05$ and $p<0.01$, respectively.

756 


\section{References}

7581 Flotho, A. \& Melchior, F. in Annual Review of Biochemistry, Vol 82 Vol. 82 Annual Review 759 of Biochemistry 357-385 (Annual Reviews, 2013).

7602 Liang, Y. C. et al. SUMO5, a Novel Poly-SUMO Isoform, Regulates PML Nuclear Bodies. Scientific Reports 6, 15, doi:10.1038/srep26509 (2016).

7623 Kunz, K., Piller, T. \& Muller, S. SUMO-specific proteases and isopeptidases of the SENP family at a glance. J Cell Sci 131, doi:10.1242/jcs.211904 (2018).

764

4 Mukhopadhyay, D. \& Dasso, M. Modification in reverse: the SUMO proteases. Trends in

765

766 Biochemical Sciences 32, 286-295, doi:10.1016/j.tibs.2007.05.002 (2007).

767

5 Liu, B. \& Shuai, K. Regulation of the sumoylation system in gene expression. Current Opinion in Cell Biology 20, 288-293, doi:10.1016/j.ceb.2008.03.014 (2008).

768 Sampson, D. A., Wang, M. \& Matunis, M. J. The small ubiquitin-like modifier-1 (SUMO-1) consensus sequence mediates Ubc9 binding and is essential for SURIO-1 modification. Journal of Biological Chemistry 276, 21664-21669, doi:10.1074/jbc.M100006200 (2001). Johnson, E. S. Protein modification by SUMO. Annual Review of Biochemistry 73, 355-382,

773 doi:10.1146/annurev.biochem.73.011303.074118 (2004).

774

775 Shuai, K. \& Liu, B. Regulation of gene-activation pathways by pias proteins in the immune system. Nature Reviews Immunology 5, 593-605, doi:10.1038/nri1667 (2005).

Rytinki, M. M., Kaikkonen, S., Pehkonen, P., Jaaskelainen, T. \& Palvimo, J. J. PIAS proteins: pleiotropic interactors associated with SUMO. Cellular and Molecular Life Sciences 66, 3029-3041, doi:10.1007/s00018-009-0061-z (2009).

779

780

781

11 Okubo, S. et al. NMR structure of the N-terminal domain of SUMO ligase PIAS1 and its

782

783

784

785

786

787

788

789

790 Tan, J. A. T., Song, J., Chen, Y. \& Durrin, L. K. Phosphorylation-Dependent Interaction of SATB1 and PIAS1 Directs SUMO-Regulated Caspase Cleavage of SATB1. Molecular and Cellular Biology 30, 2823-2836, doi:10.1128/mcb.01603-09 (2010).

791

792

793 interaction with tumor suppressor p53 and A/T-rich DNA oligomers. Journal of Biological Chemistry 279, 31455-31461, doi:10.1074/jbc.M403561200 (2004).

12 Kipp, M. et al. SAF-Box, a conserved protein domain that specifically recognizes scaffold attachment region DNA. Molecular and Cellular Biology 20, 7480-7489, doi:10.1128/mcb.20.20.7480-7489.2000 (2000).

13 van den Akker, E. et al. FLI-1 functionally interacts with PIASx alpha, a member of the PIAS E3 SUMO ligase family. Journal of Biological Chemistry 280, 38035-38046, doi:10.1074/jbc.M502938200 (2005).

14 Duval, D., Duval, G., Kedinger, C., Poch, O. \& Boeuf, H. The 'PINIT' motif, of a newly identified conserved domain of the PIAS protein family, is essential for nuclear retention of PIAS3L. FEBS Lett. 554, 111-118, doi:10.1016/s0014-5793(03)01116-5 (2003).

794

795

796

797

798

15 Palvimo, J. J. PIAS proteins as regulators of small ubiquitin-related modifier (SUMO) modifications and transcription. Biochemical Society Transactions 35, 1405-1408, doi:10.1042/bst0351405 (2007).

16 Kahyo, T., Nishida, T. \& Yasuda, H. Involvement of PIAS1 in the sumoylation of tumor suppressor p53. Molecular Cell 8, 713-718, doi:10.1016/s1097-2765(01)00349-5 (2001).

799

17 Gross, M. et al. Distinct effects of PIAS proteins on androgen-mediated gene activation in prostate cancer cells. Oncogene 20, 3880-3887, doi:10.1038/sj.onc.1204489 (2001). 
80018 Megidish, T., Xu, J. H. \& Xu, C. W. Activation of p53 by protein inhibitor of activated Stat1 (PIAS1). Journal of Biological Chemistry 277, 8255-8259, doi:10.1074/jbc.C2000001200 (2002).

803

19 Liu, B. et al. Inhibition of Stat1-mediated gene activation by PIAS1. Proceedings of the National Academy of Sciences of the United States of America 95, 10626-10631, doi:10.1073/pnas.95.18.10626 (1998).

20 Driscoll, J. J. et al. The sumoylation pathway is dysregulated in multiple myeloma and is associated with adverse patient outcome. Blood 115, 2827-2834, doi:10.1182/blood2009-03-211045 (2010).

810

811

812

21 Hoefer, J. et al. PIAS1 Is Increased in Human Prostate Cancer and Enhances Proliferation

22 Puhr, M. et al. PIAS1 is a determinant of poor survival and acts as a positive feedback regulator of AR signaling through enhanced AR stabilization in prostate cancer. Oncogene 35, 2322-2332, doi:10.1038/onc.2015.292 (2016).

818 Rabellino, A. et al. PIAS1 Promotes Lymphomagenesis through MYC Upregulation. Cell Reports 15, 2266-2278, doi:10.1016/j.celrep.2016.05.015 (2016).

24 Kadare, G. et al. PIAS1-mediated sumoylation of focal adhesion kinase activates its autophosphorylation. Journal of Biological Chemistry 278, 47434-47440, doi:10.1074/jbc.M308562200 (2003).

821

25 Streich, F. C. \& Lima, C. D. Capturing a substrate in an activated RING E3/E2-SUMO complex. Nature 536, 304-+, doi:10.1038/nature19071 (2016).

823

824 Rabellino, A. et al. The SUMO E3-ligase PIAS1 Regulates the Tumor Suppressor PML and Its Oncogenic Counterpart PML-RARA. Cancer Res. 72, 2275-2284, doi:10.1158/00085472.can-11-3159 (2012).

825

27 Lamoliatte, F., McManus, F. P., Maarifi, G., Chelbi-Alix, M. K. \& Thibault, P. Uncovering the SUMOylation and ubiquitylation crosstalk in human cells using sequential peptide immunopurification. Nature Communications 8, 11, doi:10.1038/ncomms14109 (2017). Hendriks, I. A. et al. Uncovering global SUMOylation signaling networks in a site-specific manner. Nature Structural \& Molecular Biology 21, 927-936, doi:10.1038/nsmb.2890 (2014). hypersumoylation alters filament dynamics and is a marker for human liver disease and keratin mutation. J Biol Chem 286, 2273-2284, doi:10.1074/jbc.M110.171314 (2011). Goodman, S. R. Medical cell biology. (Academic Press, 2007).

836 Hochstrasser, M. SP-RING for SUMO: new functions bloom for a ubiquitin-like protein. Cell 107, 5-8 (2001). Constituent Promyelocytic Leukemia Nuclear Body Protein That Contributes to the Intrinsic Antiviral Immune Response to Herpes Simplex Virus 1. Journal of Virology 90, 5939-5952, doi:10.1128/jvi.00426-16 (2016).

842

843

33 Lane, E. B., Hogan, B. L. M., Kurkinen, M. \& Garrels, J. I. CO-EXPRESSION OF VIMENTIN AND CYTOKERATINS IN PARIETAL ENDODERM CELLS OF EARLY MOUSE EMBRYO. Nature 303, 701-704, doi:10.1038/303701a0 (1983). 
84434 Ramaekers, F. C. S. et al. COEXPRESSION OF KERATIN-TYPE AND VIMENTIN-TYPE INTERMEDIATE FILAMENTS IN HUMAN METASTATIC CARCINOMA-CELLS. Proceedings of the National Academy of Sciences of the United States of America-Biological Sciences 80, 2618-2622, doi:10.1073/pnas.80.9.2618 (1983).

35 Thomas, J. T., Hubert, W. G., Ruesch, M. N. \& Laimins, L. A. Human papillomavirus type 31 oncoproteins E6 and E7 are required for the maintenance of episomes during the viral life

851 cycle in normal human keratinocytes. Proceedings of the National Academy of Sciences of the United States of America 96, 8449-8454, doi:10.1073/pnas.96.15.8449 (1999).

36 Anastasi, E. et al. Expression of Reg and cytokeratin 20 during ductal cell differentiation and proliferation in a mouse model of autoimmune diabetes. European Journal of Endocrinology 141, 644-652, doi:10.1530/eje.0.1410644 (1999).

37 Chu, Y. W., Runyan, R. B., Oshima, R. G. \& Hendrix, M. J. C. EXPRESSION OF COMPLETE KERATIN FILAMENTS IN MOUSE L-CELLS AUGMENTS CELL-MIGRATION AND INVASION. Proceedings of the National Academy of Sciences of the United States of America 90, 42614265, doi:10.1073/pnas.90.9.4261 (1993).

860

861

862

38 Bordeleau, F., Bessard, J., Sheng, Y. \& Marceau, N. Keratin contribution to cellular

863

864

865 mechanical stress response at focal adhesions as assayed by laser tweezers. Biochemistry and Cell Biology-Biochimie Et Biologie Cellulaire 86, 352-359, doi:10.1139/o08-076 (2008).

$39 \mathrm{Hu}, \mathrm{G}$. , Jia, F., Gao, N. \& Han, Y. Impacts of CyhospitalclinE downstream vimentin on proliferation, invasion and apoptosis of hepatoma HepG2 cell. International Journal of Clinical and Experimental Medicine 11, 5564-5571 (2018).

40 Snider, N. T. \& Omary, M. B. Post-translational modifications of intermediate filament proteins: mechanisms and functions. Nature Reviews Molecular Cell Biology 15, 163-177, doi:10.1038/nrm3753 (2014).

4 Zhu, Q. S. et al. Vimentin is a novel AKT1 target mediating motility and invasion. Oncogene 30, 457-470, doi:10.1038/onc.2010.421 (2011).

42 Snider, N. T., Weerasinghe, S. V. W., Iniguez-Lluhi, J. A., Herrmann, H. \& Omary, M. B. Keratin Hypersumoylation Alters Filament Dynamics and Is a Marker for Human Liver Disease and Keratin Mutation. Journal of Biological Chemistry 286, 2273-2284, doi:10.1074/jbc.M110.171314 (2011).

43 Goldman, R. D., Cleland, M. M., Murthy, S. N. P., Mahammad, S. \& Kuczmarski, E. R. Inroads into the structure and function of intermediate filament networks. Journal of Structural Biology 177, 14-23, doi:10.1016/j.jsb.2011.11.017 (2012).

44 Helfand, B. T. et al. Vimentin organization modulates the formation of lamellipodia. Molecular Biology of the Cell 22, 1274-1289, doi:10.1091/mbc.E10-08-0699 (2011).

880

881

882

45 Perez-Sala, D. et al. Vimentin filament organization and stress sensing depend on its single cysteine residue and zinc binding. Nature Communications 6, doi:10.1038/ncomms8287 (2015).

883

884

885

46 Satelli, A. \& Li, S. L. Vimentin in cancer and its potential as a molecular target for cancer therapy. Cellular and Molecular Life Sciences 68, 3033-3046, doi:10.1007/s00018-0110735-1 (2011).

886

887

47 Eriksson, J. E. et al. Specific in vivo phosphorylation sites determine the assembly dynamics of vimentin intermediate filaments. Journal of Cell Science 117, 919-932, doi:10.1242/jcs.00906 (2004). 
888

889

890

891

892

893

894

895

896

897

898

899

900

901

902

903

904

905

906

907

908

909

910

911

912

913

914

915

916

917

918

919

920

921

922

923

924

925

926

$927 \quad 60$

928

929

48 Robert, A., Rossow, M. J., Hookway, C., Adam, S. A. \& Gelfand, V. I. Vimentin filament precursors exchange subunits in an ATP-dependent manner. Proc. Natl. Acad. Sci. U. S. A. 112, E3505-E3514, doi:10.1073/pnas.1505303112 (2015).

49 Premchandar, A. et al. Structural Dynamics of the Vimentin Coiled-coil Contact Regions Involved in Filament Assembly as Revealed by Hydrogen-Deuterium Exchange. Journal of Biological Chemistry 291, 24931-24950, doi:10.1074/jbc.M116.748145 (2016).

50 Chou, Y. H., Khuon, S., Herrmann, H. \& Goldman, R. D. Nestin promotes the phosphorylation-dependent disassembly of vimentin intermediate filaments during mitosis. Molecular Biology of the Cell 14, 1468-1478, doi:10.1091/mbc.E02-08-0545 (2003).

51 Chen, P. et al. Protein inhibitor of activated STAT-1 is downregulated in gastric cancer tissue and involved in cell metastasis. Oncology Reports 28, 2149-2155, doi:10.3892/or.2012.2030 (2012).

52 Suman, P. et al. AP-1 Transcription Factors, Mucin-Type Molecules and MMPs Regulate the IL-11 Mediated Invasiveness of JEG-3 and HTR-8/SVneo Trophoblastic Cells. Plos One 7, 12, doi:10.1371/journal.pone.0029745 (2012).

53 Cuijpers, S. A. G., Willemstein, E. \& Vertegaal, A. C. O. Converging Small Ubiquitin-like Modifier (SUMO) and Ubiquitin Signaling: Improved Methodology Identifies Co-modified Target Proteins. Molecular \& Cellular Proteomics 16, 2281-2295, doi:10.1074/mcp.TIR117.000152 (2017).

54 Kumar, R., Gonzalez-Prieto, R., Xiao, Z. Y., Verlaan-de Vries, M. \& Vertegaal, A. C. O. The STUbL RNF4 regulates protein group SUMOylation by targeting the SUMO conjugation machinery. Nature Communications 8, doi:10.1038/s41467-017-01900-x (2017).

55 Cox, J. \& Mann, M. MaxQuant enables high peptide identification rates, individualized p.p.b.-range mass accuracies and proteome-wide protein quantification. Nature Biotechnology 26, 1367-1372, doi:10.1038/nbt.1511 (2008).

56 Cox, J. et al. Andromeda: A Peptide Search Engine Integrated into the MaxQuant Environment. Journal of Proteome Research 10, 1794-1805, doi:10.1021/pr101065j (2011).

57 Feuermann, M., Gaudet, P., Mi, H. Y., Lewis, S. E. \& Thomas, P. D. Large-scale inference of gene function through phylogenetic annotation of Gene Ontology terms: case study of the apoptosis and autophagy cellular processes. Database-the Journal of Biological Databases and Curation, 11, doi:10.1093/database/baw155 (2016).

$58 \mathrm{Mi}, \mathrm{H}$. Y. et al. PANTHER version 11: expanded annotation data from Gene Ontology and Reactome pathways, and data analysis tool enhancements. Nucleic Acids Research 45, D183-D189, doi:10.1093/nar/gkw1138 (2017).

59 Huang, D. W., Sherman, B. T. \& Lempicki, R. A. Bioinformatics enrichment tools: paths toward the comprehensive functional analysis of large gene lists. Nucleic Acids Research 37, 1-13, doi:10.1093/nar/gkn923 (2009).

60 Colaert, N., Helsens, K., Martens, L., Vandekerckhove, J. \& Gevaert, K. Improved visualization of protein consensus sequences by iceLogo. Nature Methods 6, 786-787, doi:10.1038/nmeth1109-786 (2009). 
93061 Petersen, B., Petersen, T. N., Andersen, P., Nielsen, M. \& Lundegaard, C. A generic method 931 for assignment of reliability scores applied to solvent accessibility predictions. Bmc Structural Biology 9, 10, doi:10.1186/1472-6807-9-51 (2009).

934

62 von Mering, C. et al. STRING: a database of predicted functional associations between

935 proteins. Nucleic Acids Research 31, 258-261, doi:10.1093/nar/gkg034 (2003).

936

937

938

939

940

941

63 Szklarczyk, D. et al. The STRING database in 2011: functional interaction networks of proteins, globally integrated and scored. Nucleic Acids Research 39, D561-D568, doi:10.1093/nar/gkq973 (2011).

64 Shannon, P. et al. Cytoscape: A software environment for integrated models of biomolecular interaction networks. Genome Research 13, 2498-2504, doi:10.1101/gr.1239303 (2003).

942

65 Cline, M. S. et al. Integration of biological networks and gene expression data using

943 Cytoscape. Nature Protocols 2, 2366-2382, doi:10.1038/nprot.2007.324 (2007).

944

66 McManus, F. P., Lamoliatte, F. \& Thibault, P. Identification of cross talk between

945 SUMOylation and ubiquitylation using a sequential peptide immunopurification approach. Nature protocols 12, 2342-2358, doi:10.1038/nprot.2017.105 (2017). 